Supramolecular Catalysis of Acyl Transfer within $\mathrm{Zn}-$ Porphyrin-Based Metal-Organic Cages

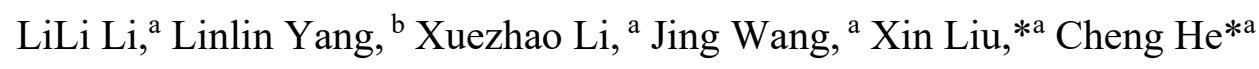

${ }^{a}$ State Key Laboratory of Fine Chemicals, Dalian University of Technology, Dalian,

116023, China; ${ }^{b}$ Xinxiang Key Laboratory of Forensic Science Evidence, School of

Forensic Medicine, Xinxiang Medical University, Xinxiang, 453003, China.

*Correspondence authors.

E-mail: hecheng@dlut.edu.cn

E-mail: xliu@dlut.edu.cn 


\section{Measurements and Materials}

1.1 Materials. $\mathrm{Zn}(\mathrm{II})$ 5,10,15,20-tetrakis(4-aminophenyl) porphine (Zn-TAPP), ${ }^{1}$ 5methyl-2,2'-bipyridine, ${ }^{2} \quad$ 2,2'-bipyridinyl-5-carboxylic acid, ${ }^{2} \quad$ 2,2'-bipyridinyl-5carboxyl chloride, ${ }^{2}$ N,N-dimethyl-6-phenylpiconlinamide, ${ }^{3}$ (6-phenylpyridin-2y)methanol ${ }^{4}$ and 1-(1H-imidazol-1-yl)-3-methyl-1-butanone ${ }^{5}$ were synthesized according to published procedures. Methacrolein (Energy Chemical), zinc bis(trifluoromethane sulfonimide) $\quad\left(\mathrm{Zn}\left(\mathrm{NTf}_{2}\right)_{2}\right) \quad(\mathrm{TCI}), \quad$ 2-hydroxymethyl-5bromopyridine (Energy Chemical), phenylboronic acid (Energy Chemical), 4methoxyphenylboronic acid (Energy Chemical), 4-biphenylboronic acid (Energy Chemical), 6-phenylpyridine-2-carboxylic acid (Energy Chemical), 2-PC (Energy Chemical), 3-PC (Energy Chemical), 4-PC (Energy Chemical), $\mathrm{N}$-acetylimidazole (NAI) (Energy Chemical), $\mathrm{Me}_{2} \mathrm{NH}$ (Aladdin), $\mathrm{NaH}$ (Aladdin), $\mathrm{ZnI}_{2}$ (Aladdin), 3methylbutyric acid (Energy Chemical), and N, N'-carbonyldiimidazole (Energy Chemical) were obtained from commercial sources and used as received. Solvents were obtained either from Sinopharm chemical or Tianjin Kermel chemical and used as received.

1.2 Measurements. All chemicals were of reagent grade quality obtained from commercial sources and used as supplied unless otherwise stated. ${ }^{1} \mathrm{H}$ NMR spectra was measured on a Varian DLG 400M and Bruker Avance 500M spectrometer. ESI mass spectra were carried out on a HPLC-Q-Tof MS spectrometer. UV-vis spectra were measured on a HP 8453 spectrometer, and the data obtained from the ultraviolet-visible titration were then fitted using nonlinear analysis with DynaFit programme ${ }^{6}$ (Biokin Software) to the binding equation derived for either 1:1 model. The error reported for each affinity value is the formal standard error calculated by DynaFit ${ }^{6}$ as the square root of the diagonal elements of the variance-covariance matrix. Gas chromatography analysis was performed on an Agilent 6890N instrument equipped with an HP-5 capillary column $(30 \mathrm{~m} \times 0.32 \mathrm{~mm} \times 0.25 \mu \mathrm{m})$ and FID detector.

\section{Preparation and characterizations.}




\subsection{Synthesis of ligands}
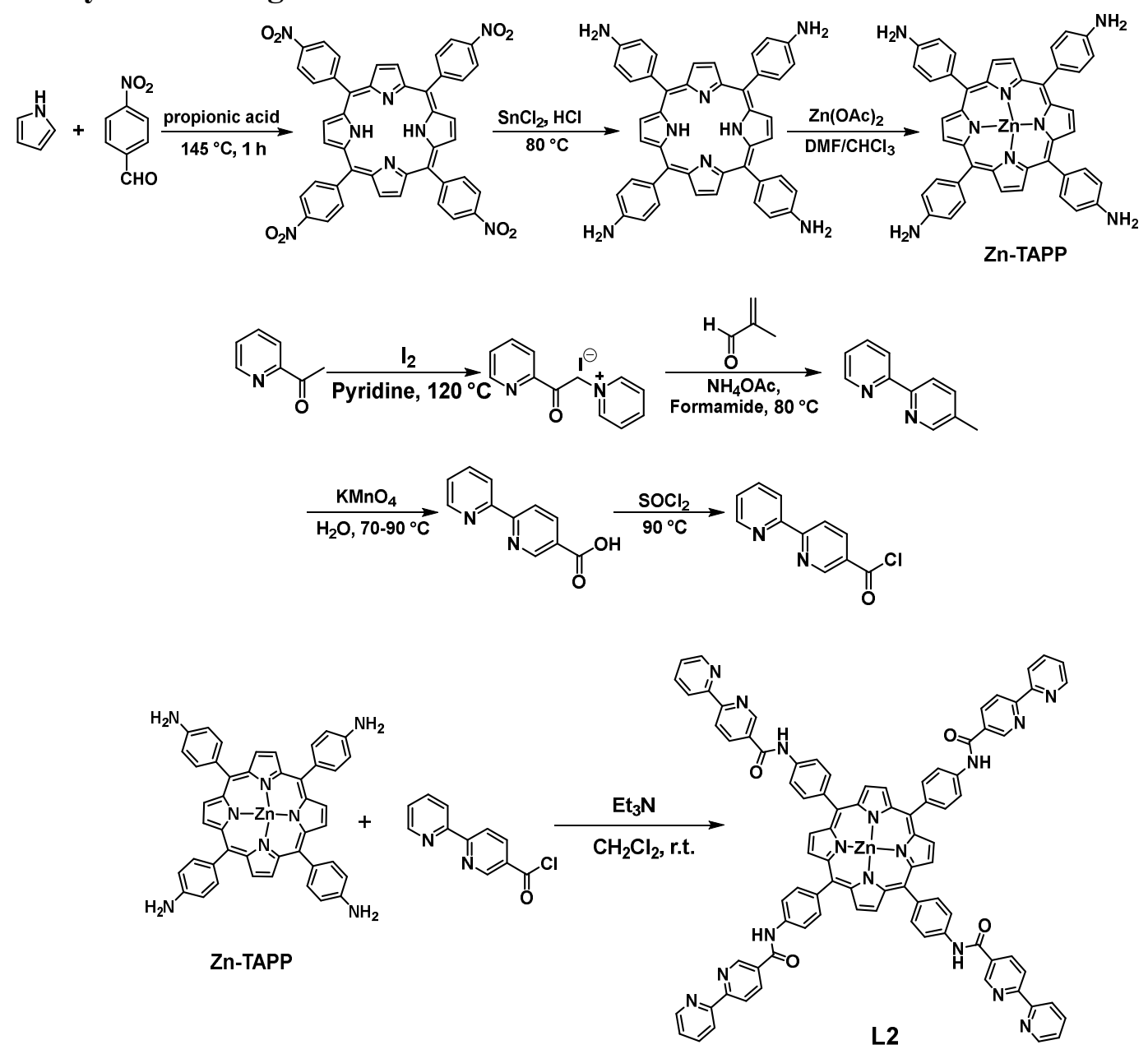

Scheme S1. The synthetic routes of the ligands.

Zn(II) 5,10,15,20-tetrakis(4-aminophenyl) porphine $\left(\right.$ Zn-TAPP). ${ }^{1} \quad 5,10,15,20$ Tetrakis(4-aminophenyl)-21H, 23H-porphine (TAPP) (354 mg, $0.52 \mathrm{mmol}, 1$ equiv.) was dissolved in a 1:3 mixture of $\mathrm{DMF}$ and $\mathrm{CHCl}_{3}(40 \mathrm{~mL}$ in total). A MeOH solution $(10 \mathrm{~mL})$ of zinc acetate dihydrate $(455 \mathrm{mg}, 2.08 \mathrm{mmol}, 4$ equiv.) was then added. The resulting mixture was stirred for $24 \mathrm{~h}$ at $80{ }^{\circ} \mathrm{C}$. After cooling to room temperature, the mixture was added to $200 \mathrm{~mL}$ water. After being settled down, the mixture was filtered. The purple solid was collected and dried under vacuum to give the product $\mathbf{Z n - T A P P}$ (325 mg, yield 82\%).

${ }^{1}$ H NMR (DMSO-d $\boldsymbol{d}^{\mathbf{6}}, \mathbf{4 0 0}$ MHz): $\delta 8.85(8 \mathrm{H}, \mathrm{s}), 7.82(8 \mathrm{H}, \mathrm{d}, \mathrm{J}=8 \mathrm{~Hz}), 6.98(8 \mathrm{H}, \mathrm{d}, \mathrm{J}=8$ $\mathrm{Hz}), 5.54(8 \mathrm{H}, \mathrm{s})$.

5-Methyl-2,2'-bipyridine. 2-acetylpyridine (5.6 mL, $50.0 \mathrm{mmol})$ and $\mathrm{I}_{2}(14.2 \mathrm{~g}, 56.0$ 
mmol) were dissolved in pyridine $(60 \mathrm{~mL})$ and refluxed for 6 hours. The reaction mixture was then cooled to room temperature and the resulting suspension was filtered, and washed with ethyl ether. The pure 1-(2-pyridylacetyl) pyridinium iodide was obtained after re-crystallization from a saturated EtOH solution and used subsequently. Methacrolein $(1.8 \mathrm{~mL}, 22.0 \mathrm{mmol})$ and $\mathrm{NH}_{4} \mathrm{OAc}(9.3 \mathrm{~g}, 120.0 \mathrm{mmol})$ were sequentially added to the solution of 1-(2-pyridylacetyl) pyridinium iodide (6.5 g, $20 \mathrm{mmol})$ in formamide $(200 \mathrm{~mL})$. The mixture was stirred at $80^{\circ} \mathrm{C}$ for $16 \mathrm{~h}$. At this time, the crude mixture was cooled and extracted with diethyl ether $(3 \times 200 \mathrm{~mL})$. The combined organic layers were washed with brine $(200 \mathrm{~mL})$, dried over $\mathrm{MgSO}_{4}$, filtered, and concentrated under reduced pressure. The crude product was purified by column chromatography $\left(\mathrm{CH}_{2} \mathrm{Cl}_{2}: \mathrm{MeOH}, 95: 5 \mathrm{v} / \mathrm{v}\right)$ to yield 5-Methyl-2,2'-bipyridine $(3.0 \mathrm{~g}$, $80 \%)$ as a brown oil.

${ }^{1}$ H NMR (CDCl 3,400 MHz): $\delta=8.61(1 \mathrm{H}, \mathrm{d}, \mathrm{J}=4.8 \mathrm{~Hz}), 8.44(1 \mathrm{H}, \mathrm{s}), 8.27(1 \mathrm{H}, \mathrm{d}$, $\mathrm{J}=8.0 \mathrm{~Hz}), 8.20(1 \mathrm{H}, \mathrm{d}, \mathrm{J}=8.0 \mathrm{~Hz}), 7.70-7.74(1 \mathrm{H}, \mathrm{td}, \mathrm{J}=8.0,1.6 \mathrm{~Hz}), 7.52-7.55(1 \mathrm{H}, \mathrm{dd}$, $\mathrm{J}=8.0,2.0 \mathrm{~Hz}), 7.18-7.21(1 \mathrm{H}, \mathrm{m}), 2.30(3 \mathrm{H}, \mathrm{s})$.

2,2'-Bipyridinyl-5-carboxylic acid. Potassium permanganate $(12.3 \mathrm{~g}, 78 \mathrm{mmol})$ was added in 7 portions at $1 \mathrm{~h}$ intervals to a solution of 5-Methyl-2,2'-bipyridine (3.4 g, 20 mmol) in water $(200 \mathrm{~mL})$. The mixture was heated at $70{ }^{\circ} \mathrm{C}$ for $3 \mathrm{~h}$ and then at $90{ }^{\circ} \mathrm{C}$ for $4 \mathrm{~h}$ more. The brown mixture was then filtered while hot through celite and washed with hot water $(2 \times 25 \mathrm{~mL})$. The filtrate was concentrated to approximately $10 \mathrm{~mL}$ under reduced pressure, and then $1 \mathrm{M} \mathrm{HCl}$ was added slowly until a $\mathrm{pH}$ of 4 was obtained. The residue was then filtered and dried to obtain pure 2,2'-Bipyridinyl-5-carboxylic acid $(1.4 \mathrm{~g}, 35 \%)$ as a white solid.

${ }^{1}$ H NMR (DMSO-d ${ }^{6}, 400$ MHz): $\delta=13.5(1 \mathrm{H}, \mathrm{s}), 9.17(1 \mathrm{H}, \mathrm{d}, \mathrm{J}=1.6 \mathrm{~Hz}), 8.74(1 \mathrm{H}$, $\mathrm{d}, \mathrm{J}=4.0 \mathrm{~Hz}), 8.41-8.53(3 \mathrm{H}, \mathrm{m}), 8.0(1 \mathrm{H}, \mathrm{td}, \mathrm{J}=7.5,1.2 \mathrm{~Hz}), 7.51-7.54(1 \mathrm{H}, \mathrm{m})$.

2,2'-Bipyridinyl-5-carboxyl chloride. The 2,2'-bipyridine-5-carboxylic acid (0.8 g, 4 mmol) was added to excess thionyl chloride $(20 \mathrm{~mL})$, the resultant heterogeneous solution was refluxed until it became homogeneous. The resultant reaction mixture was evaporated to dryness and dried in vacuo to afford the title compound as a pale-yellow 
powder. The product was used for the syntheses of $\mathbf{L} \mathbf{2}$ without purification.

Zn(II)5,10,15,20-tetrayltetrakis(benzene-4,1-diyl))tetrakis(([2,2'-bipyridine]-5carboxamide) porphine (L2). To 2,2'-Bipyridinyl-5-carboxyl chloride (0.87 g, 4 mmol) in $\mathrm{CH}_{2} \mathrm{Cl}_{2}(20 \mathrm{~mL}), \mathrm{Zn}$-TAPP $(0.36 \mathrm{~g}, 0.5 \mathrm{mmol})$ in $\mathrm{CH}_{2} \mathrm{Cl}_{2}(20 \mathrm{~mL})$ was added dropwise with magnetic stirring. After an addition of triethylamine $(2.5 \mathrm{~mL})$, the mixture was continued stirred for $5 \mathrm{~h}$ at room temperature. The residue was filtered, washed with $\mathrm{CH}_{2} \mathrm{Cl}_{2}$ and $\mathrm{CH}_{3} \mathrm{OH}$ and dried in vacuo to yield $\mathbf{L 2}$ as dark purple solid (0.6 g, 80\%). ${ }^{1} \mathrm{H}$ NMR (DMSO-d ${ }^{6}, 400$ MHz): $\delta=11.02$ (4H, s), 9.39 (4H, s), 8.90 $(8 \mathrm{H}, \mathrm{s}), 8.80$ (4H, d, J=4 Hz), $8.64(8 \mathrm{H}, \mathrm{s}), 8.54$ (4H, d, J= $8 \mathrm{~Hz}), 8.22-8.29$ (16H, m), $8.05(4 \mathrm{H}, \mathrm{t}, \mathrm{J}=8 \mathrm{~Hz}), 7.56(4 \mathrm{H}, \mathrm{t}, \mathrm{J}=6.2 \mathrm{~Hz})$.

${ }^{13}$ C NMR (DMSO-d ${ }^{6}, 400$ MHz): $\delta=164.71,162.78,157.96,154.91,150.07,149.94$, $149.31,138.88,138.08,137.39,135.02,132.14,131.27,125.37,121.65,120.55,118.88$. MALDI: [L2] $]^{+} 1464.40$.

\subsection{Synthesis of the cages}
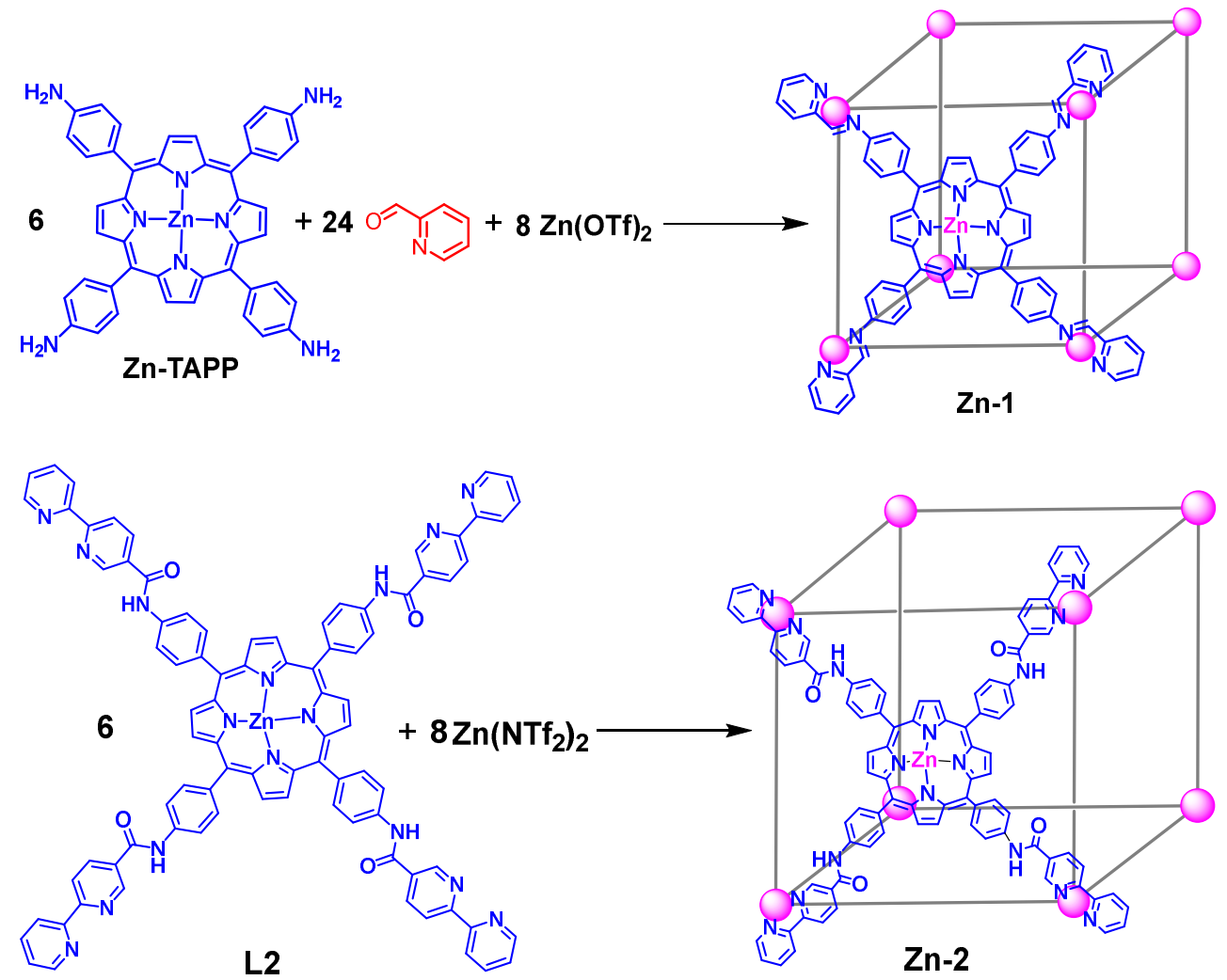

Scheme S2. The self-assembly of Zn-1 (up) and Zn-2 (down). 
Zn-1. A mixture of Zn-TAPP (73.6 mg, $0.1 \mathrm{mmol})$, 2-formylpyridine (37 $\mu \mathrm{L}, 0.4 \mathrm{mmol}$ ), and $\mathrm{Zn}(\mathrm{OTf})_{2}(48.3 \mathrm{mg}, 0.133 \mathrm{mmol})$ was dissolved in $30 \mathrm{~mL}$ DMF and then was heated at $70^{\circ} \mathrm{C}$ for $12 \mathrm{~h}$ to give a clear solution. The vapor of diethyl ether was allowed to diffuse slowly into a DMF solution of the reaction mixture. Block shaped dark purple crystals formed after one week in $76.0 \%$ yield $(120.0 \mathrm{mg})$.

${ }^{1} \mathrm{H}$ NMR (CD $\mathbf{3}$ CN/DMF-d $\boldsymbol{d}^{7}$ 3:2, $\left.500 \mathrm{MHz}\right): \delta=9.50(24 \mathrm{H}, \mathrm{d}, \mathrm{J}=10.0 \mathrm{~Hz}), 9.29(24 \mathrm{H}$, s), $8.86(24 \mathrm{H}, \mathrm{d}, \mathrm{J}=5.0 \mathrm{~Hz}), 8.67-8.63(48 \mathrm{H}, \mathrm{m}), 8.57(24 \mathrm{H}, \mathrm{d}, \mathrm{J}=5.0 \mathrm{~Hz}), 8.15(48 \mathrm{H}, \mathrm{s})$, $7.79(24 \mathrm{H}, \mathrm{d}, \mathrm{J}=5.0 \mathrm{~Hz}), 6.85(24 \mathrm{H}, \mathrm{s}), 6.38(24 \mathrm{H}, \mathrm{s})$.

${ }^{13}$ C NMR (CD 3 CN/DMF- $\boldsymbol{d}^{7}$ 3:2, 500 MHz): $\delta=166.71,162.85,150.76,150.11$, $149.87,147.64,143.49,142.73,136.59,135.79,132.27,132.07,131.77,131.65$, $123.38,120.47,120.18,119.88$.

${ }^{19}$ F NMR (CD3CN/DMF- $d^{7}$ 3:2, $\left.500 \mathrm{MHz}\right): \delta=79.00$.

Zn-2. A mixture of $\mathbf{L 2}(87.8 \mathrm{mg}, 0.06 \mathrm{mmol})$ and Znic bis(trifluoromethane sulfonimide) (50.0 mg, $0.08 \mathrm{mmol}$ ) was dissolved in $20 \mathrm{~mL}$ DMF. The reaction mixture was heated at $70{ }^{\circ} \mathrm{C}$ for 16 hours. This solution was added to diethyl ether and the product was obtained after centrifugation and then dried. The desired product was obtained as a purple solid in $85.0 \%$ yield $(117.1 \mathrm{mg})$.

${ }^{1}$ H NMR (CD $\mathbf{C N}, 500$ MHz): $\delta=9.59$ (24H, s), 9.39 (24H, s), 8.99 (48H, s), 8.96 $(24 \mathrm{H}, \mathrm{d}, \mathrm{J}=10.0 \mathrm{~Hz}), 8.92(24 \mathrm{H}, \mathrm{d}, \mathrm{J}=5.0 \mathrm{~Hz}), 8.76(24 \mathrm{H}, \mathrm{d}, \mathrm{J}=5 \mathrm{~Hz}), 8.68$ (24H, d, J=5 $\mathrm{Hz}), 8.45(24 \mathrm{H}, \mathrm{t}, \mathrm{J}=5 \mathrm{~Hz}), 8.33(48 \mathrm{H}, \mathrm{d}, \mathrm{J}=5 \mathrm{~Hz}), 8.26$ (48H, d, J=5 Hz), 7.97 (24H, t, $\mathrm{J}=5 \mathrm{~Hz})$.

${ }^{13}$ C NMR (CD 3 CN, 500 MHz): $\delta=163.24,150.84,148.80,148.15,142.82,140.31$, $140.31,138.47,135.56,134.47,132.38,128.55,124.40,124.21,123.15,119.38,116.75$. ${ }^{19}$ F NMR (CD3CN, $\left.500 \mathrm{MHz}\right): \delta=80.15$. 

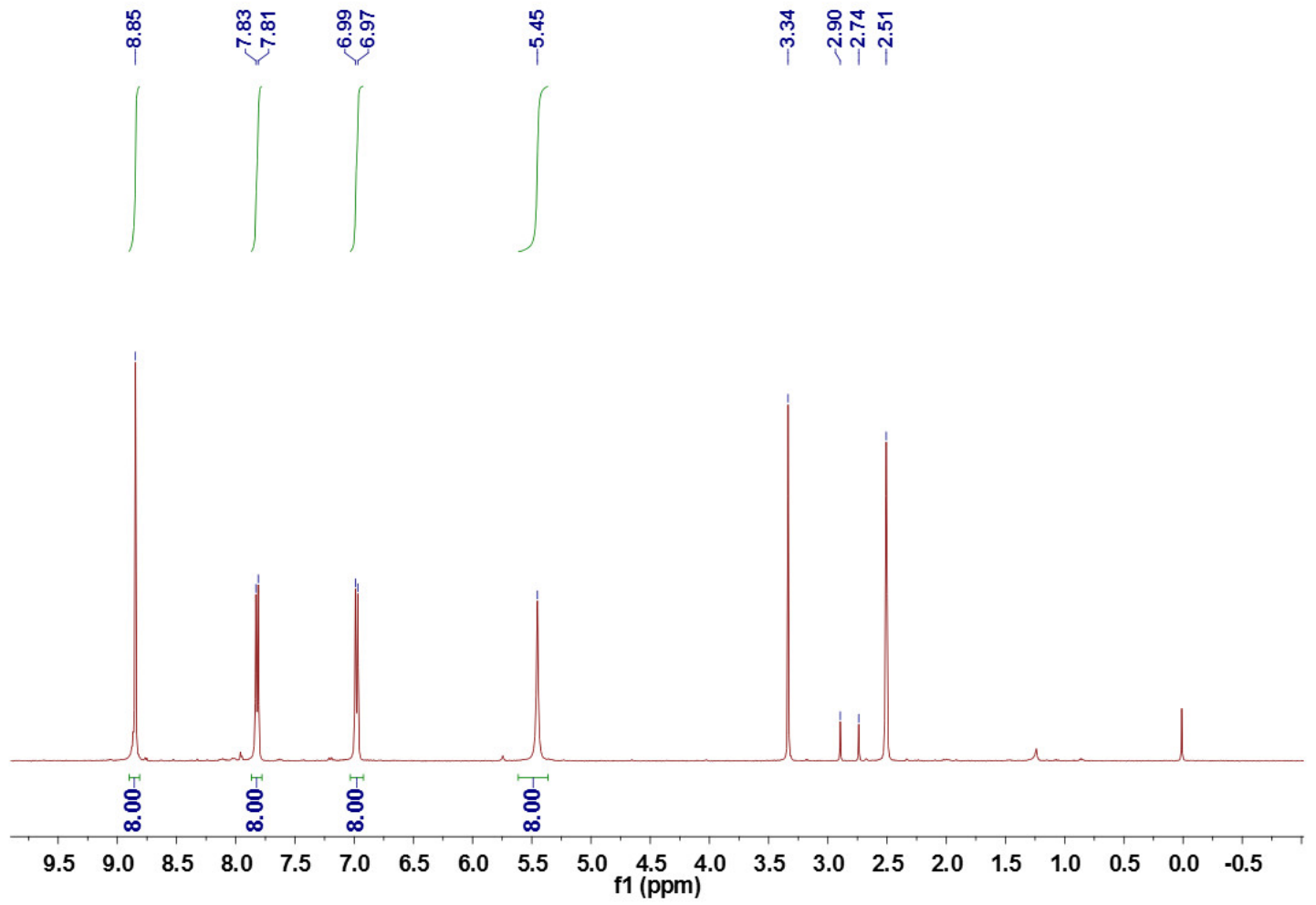

Figure S1. ${ }^{1} \mathrm{H}$ NMR spectrum $\left(400 \mathrm{MHz}\right.$, DMSO- $\left.d^{6}\right)$ of Zn-TAPP.

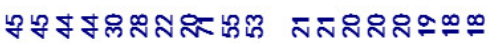

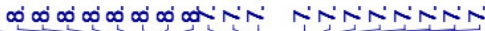
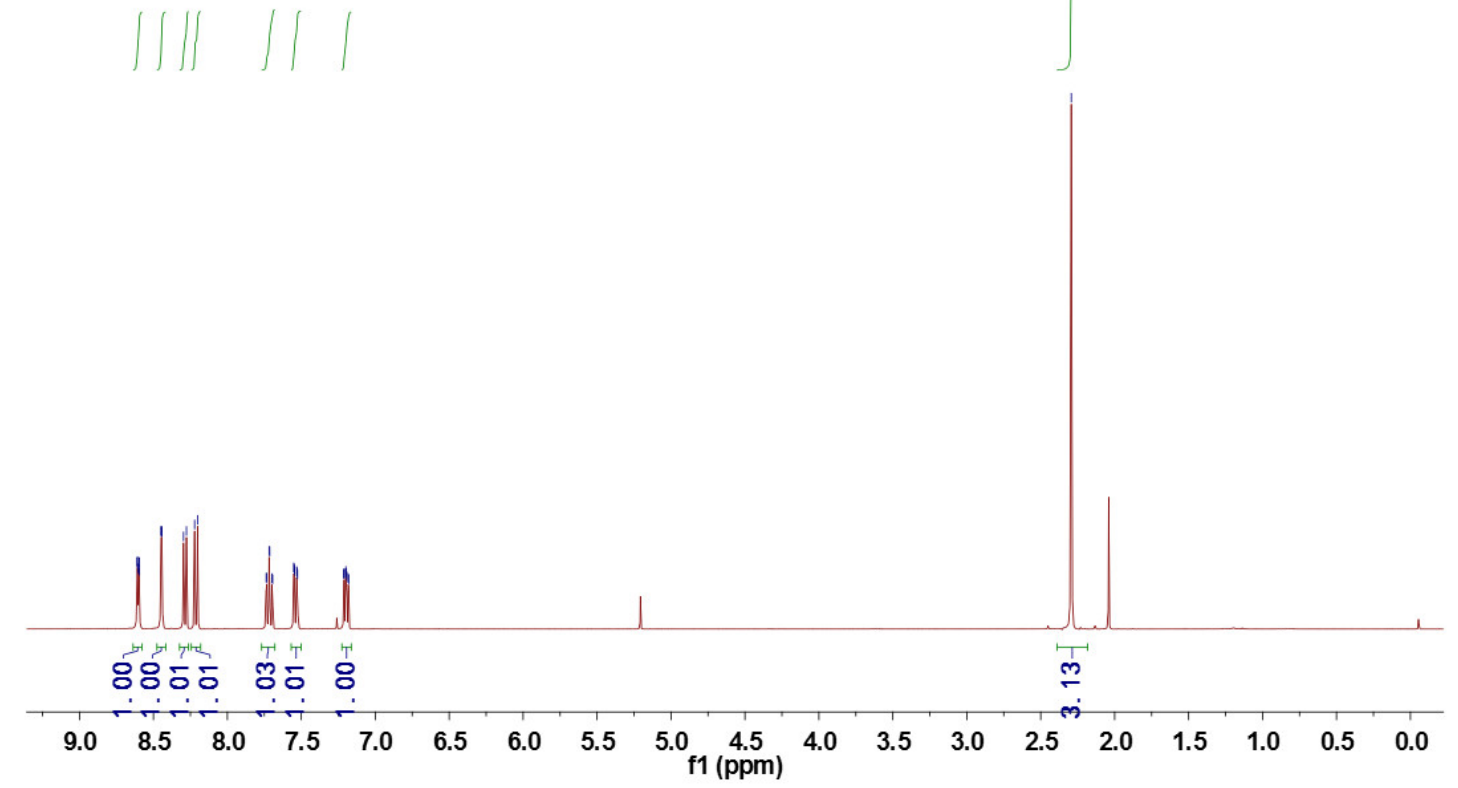

Figure S2. ${ }^{1} \mathrm{H}$ NMR spectrum (400 MHz, DMSO- $d^{6}$ ) of 5-Methyl-2,2'-bipyridine. 


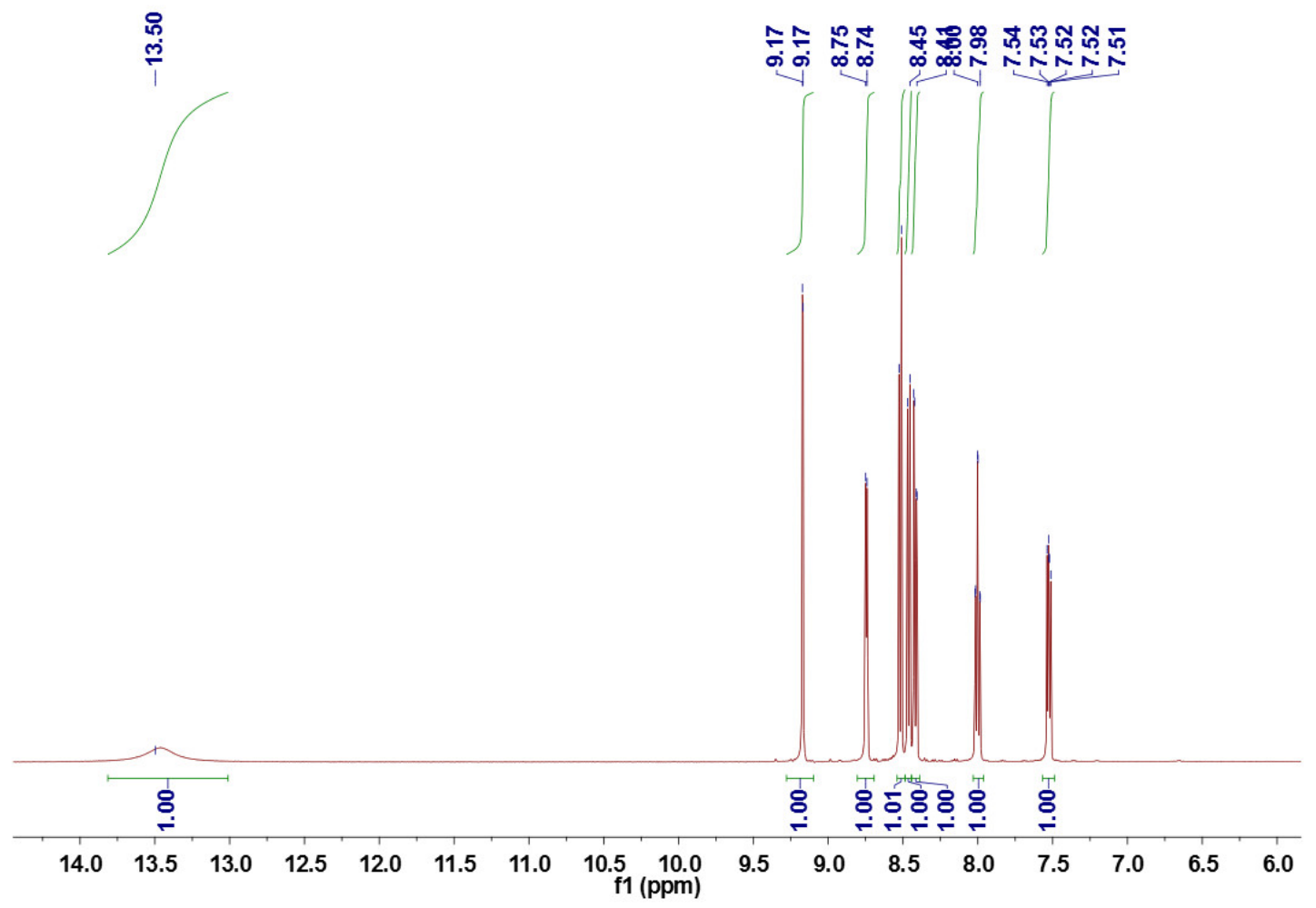

Figure S3. ${ }^{1} \mathrm{H}$ NMR spectrum (400 MHz, DMSO- $d^{6}$ ) of 2,2'-Bipyridinyl-5-carboxylic acid.
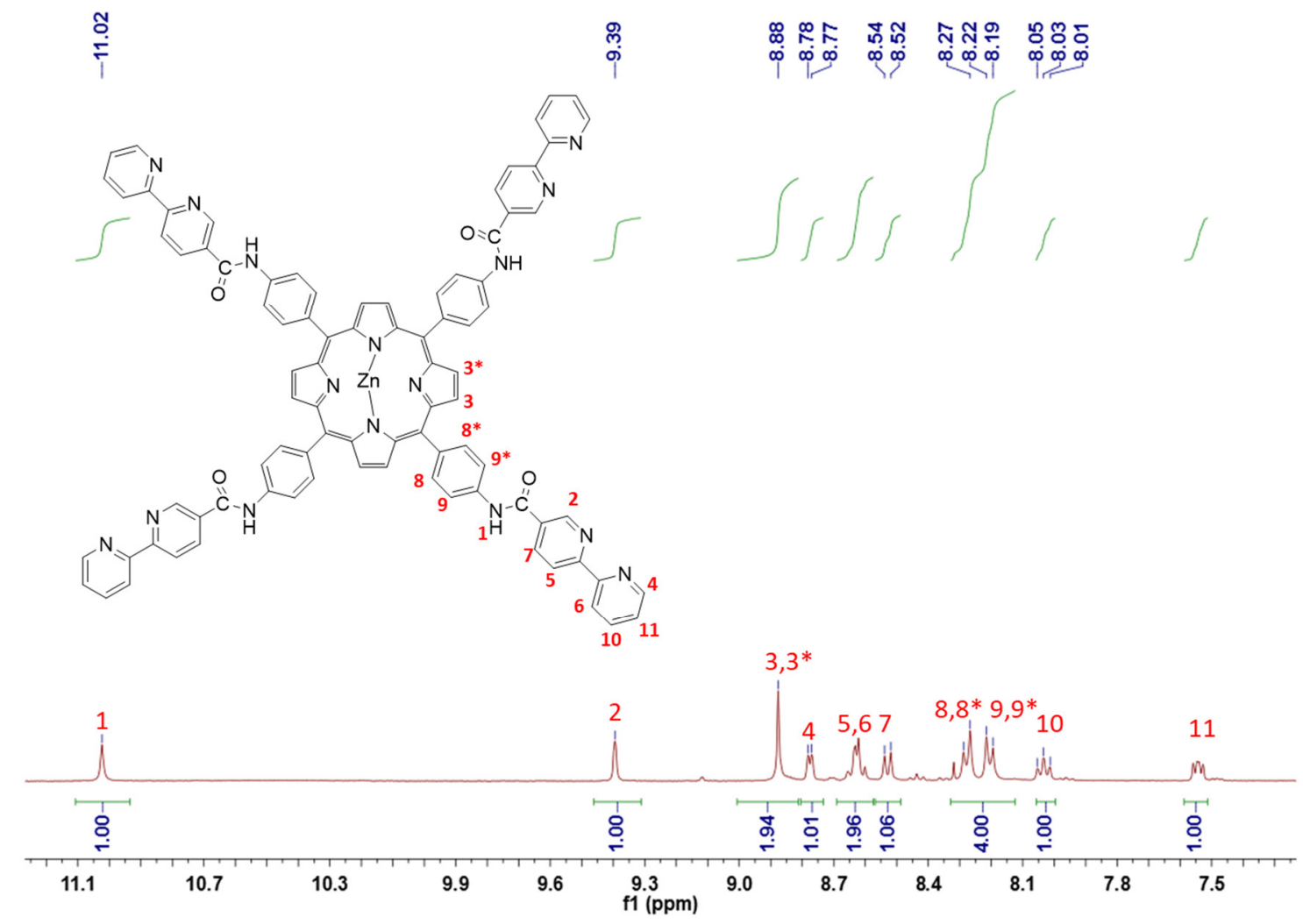

Figure S4. $\quad{ }^{1} \mathrm{H} \quad \mathrm{NMR}$ spectrum $\quad\left(400 \quad \mathrm{MHz}, \quad \mathrm{DMSO}-d^{6}\right) \quad$ of $\mathrm{Zn}(\mathrm{II}) \quad 5,10,15,20-$ tetrayltetrakis(benzene-4,1-diyl))tetrakis(([2,2'-bipyridine]-5-carboxamide) porphine. 

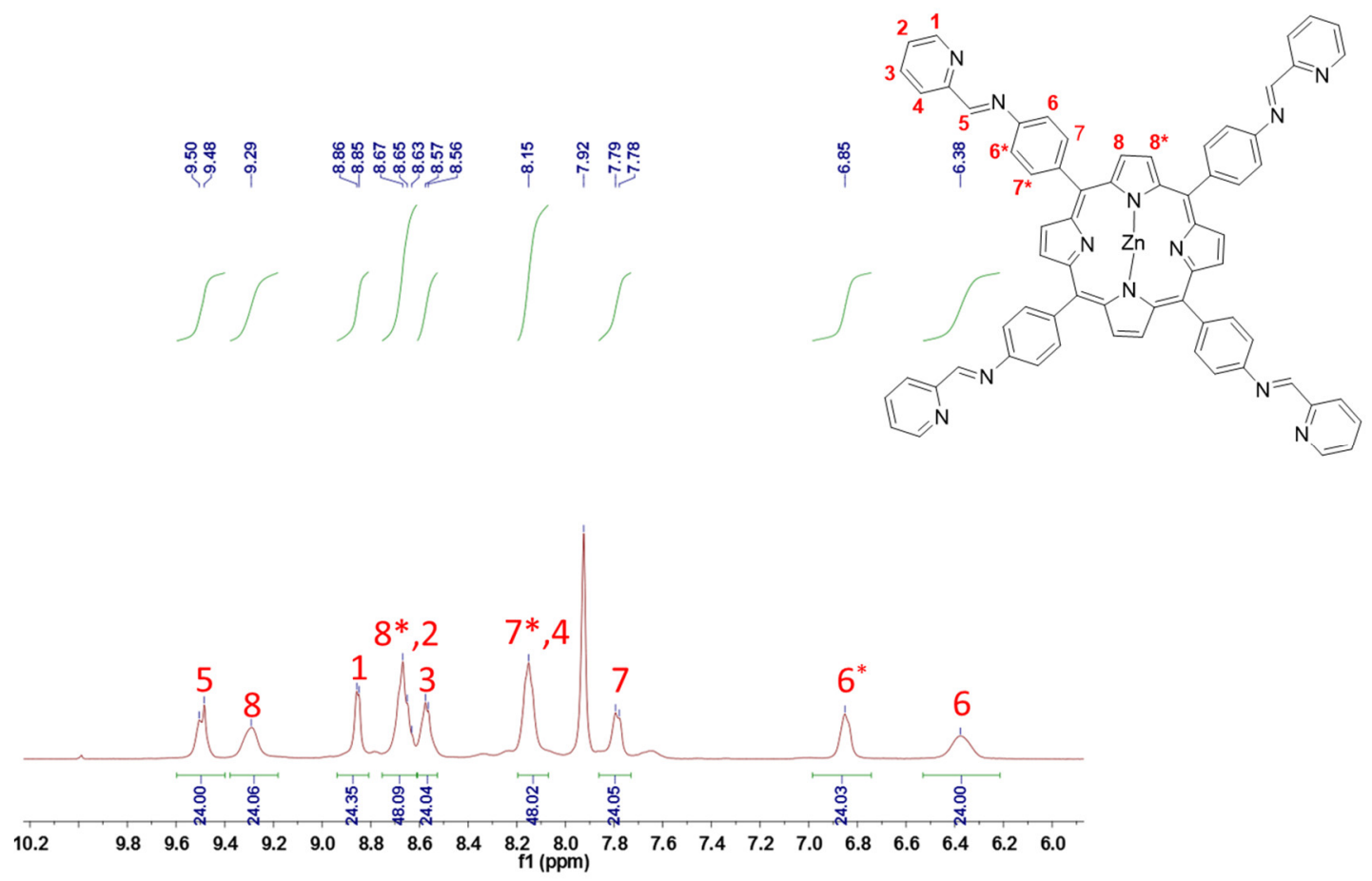

Figure S5. ${ }^{1} \mathrm{H}$ NMR spectrum for Zn-1.

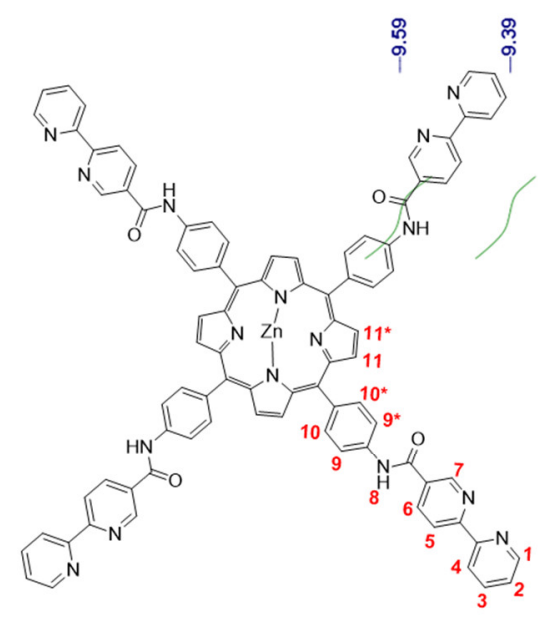

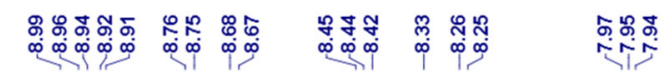

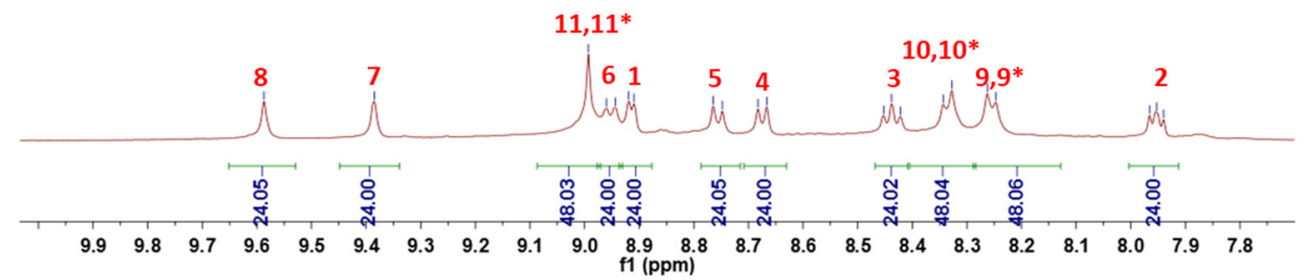

Figure S6. ${ }^{1} \mathrm{H}$ NMR spectrum for $\mathbf{Z n - 2}$. 


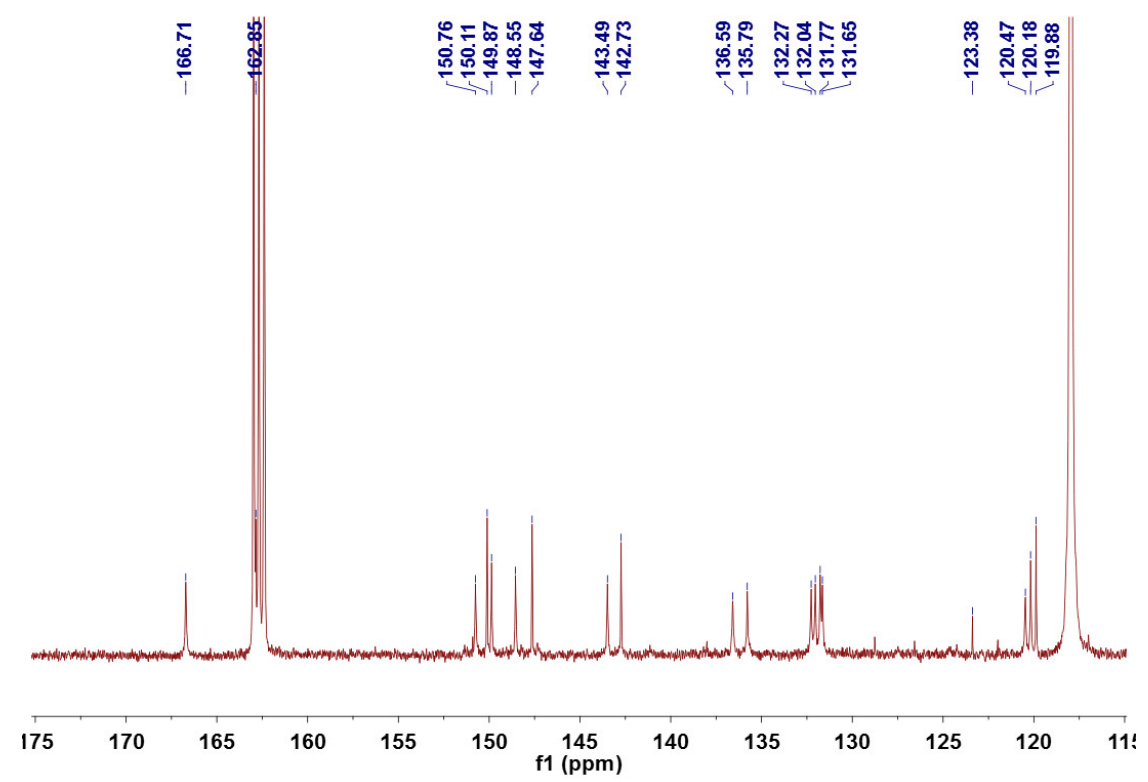

Figure S7. ${ }^{13} \mathrm{C}$ NMR spectrum for Zn-1.

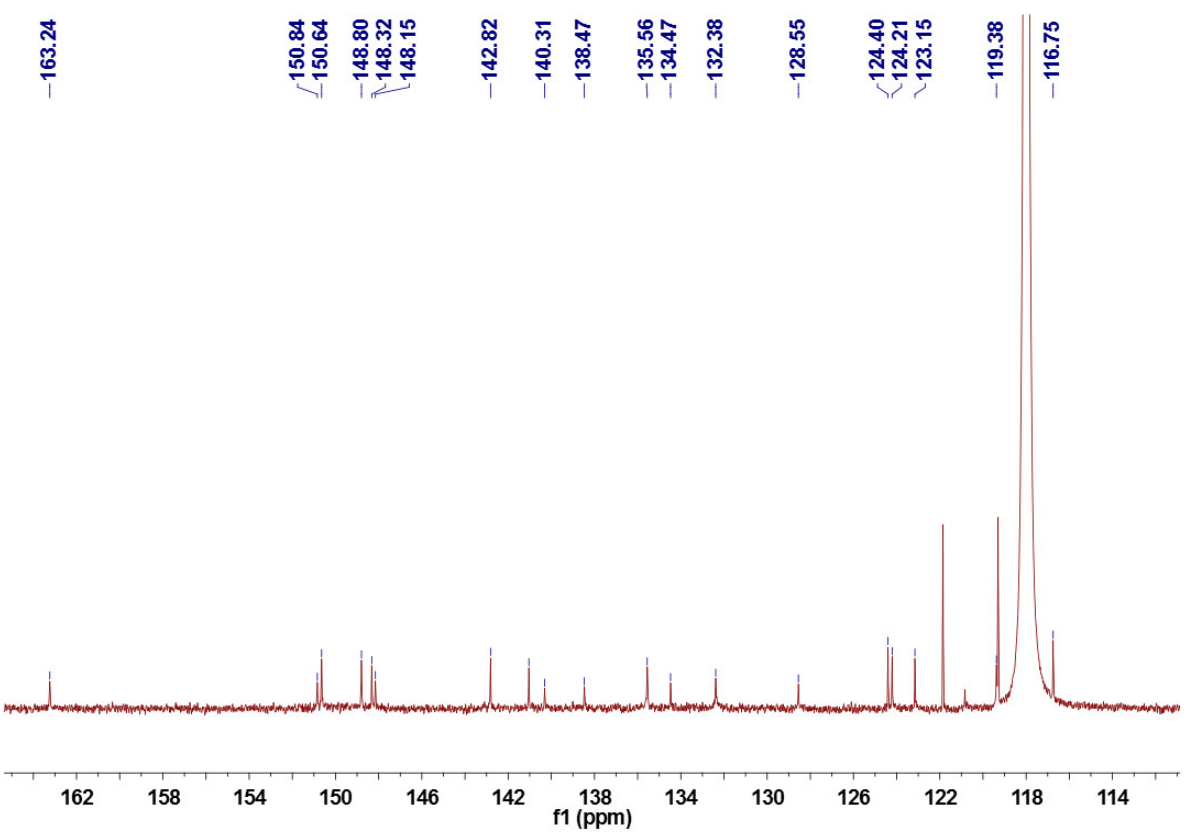

Figure S8. ${ }^{13} \mathrm{C}$ NMR spectrum for $\mathbf{Z n - 2}$. 


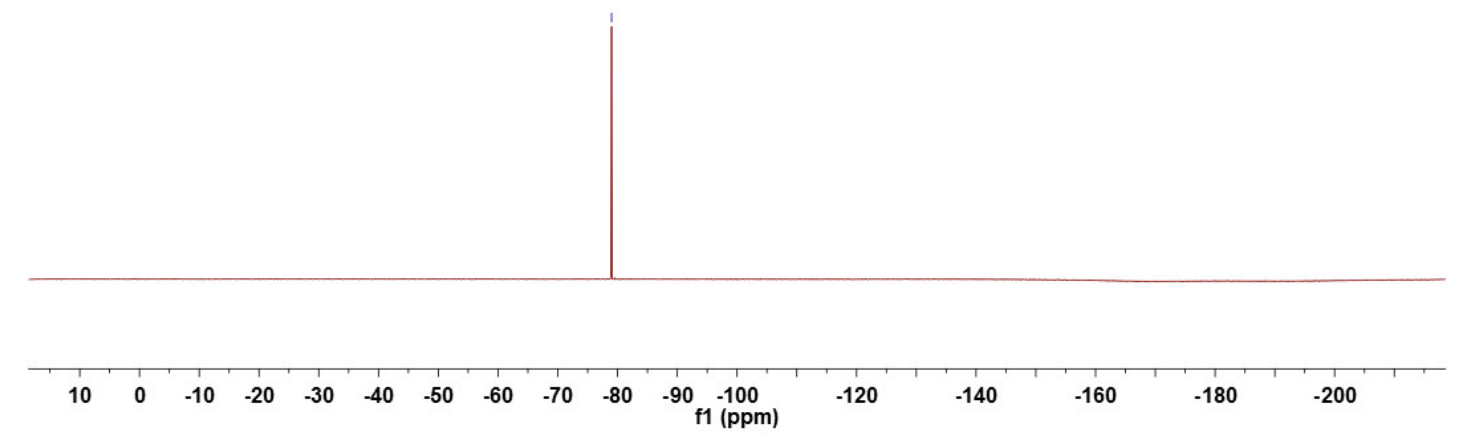

Figure S9. ${ }^{19} \mathrm{~F}$ NMR spectrum of $\mathbf{Z n - 1}$ ஸீ

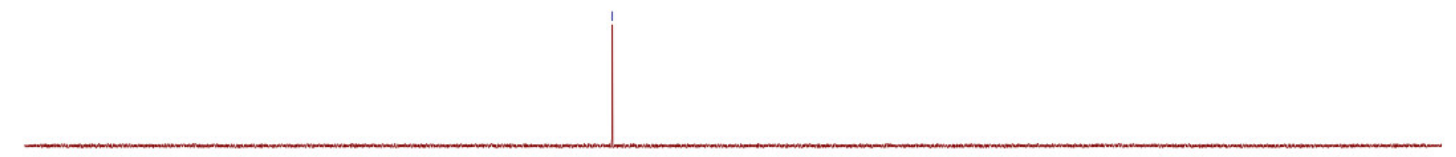

$\begin{array}{llllllllllll}10 & 0 & -10 & -20 & -30 & -40 & -50 & -60 & -70 & -80 & -90 & -100\end{array}$

\begin{tabular}{lllll|l|l|l|l|}
-120 & -140 & -160 & -180 & -200
\end{tabular}

Figure S10. ${ }^{19} \mathrm{~F}$ NMR spectrum of $\mathbf{Z n - 2}$. 


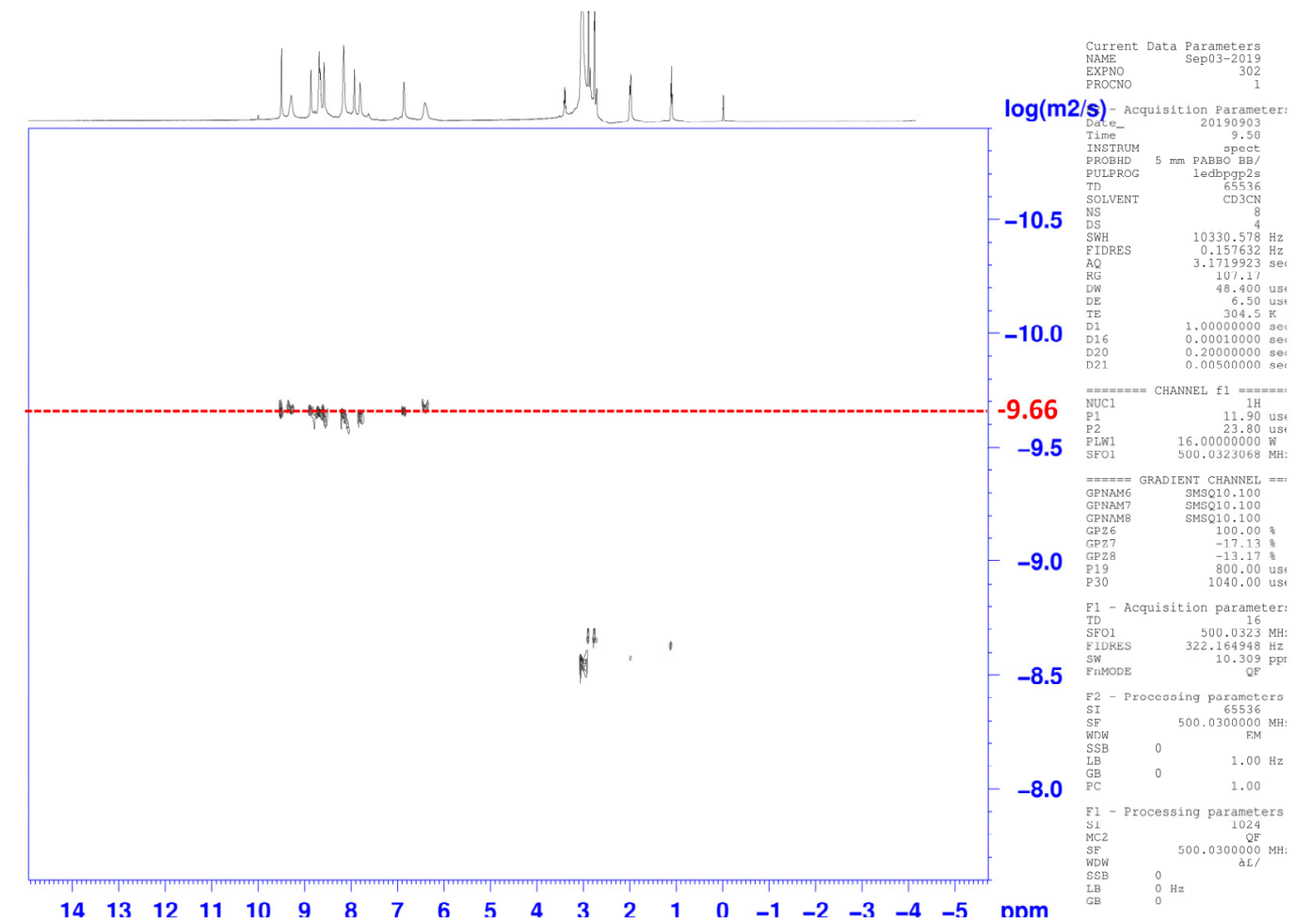

Figure S11. DOSY spectrum $(500 \mathrm{M}, 298 \mathrm{~K})$ of $\mathbf{Z n - 1}$ in $\mathrm{CD}_{3} \mathrm{CN}$ : DMF- $d^{7}=3: 2$.

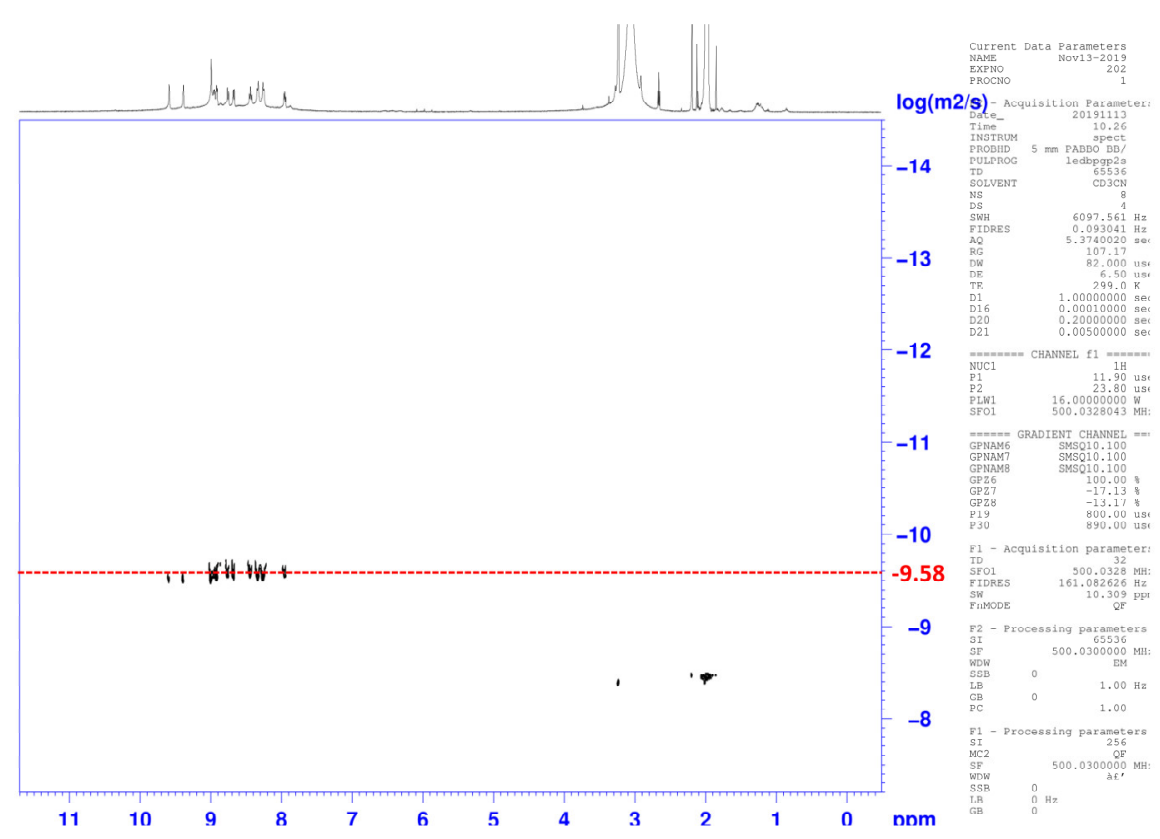

Figure S12. DOSY spectrum $(500 \mathrm{M}, 298 \mathrm{~K})$ of $\mathbf{Z n - 2}$ in $\mathrm{CD}_{3} \mathrm{CN}$. 


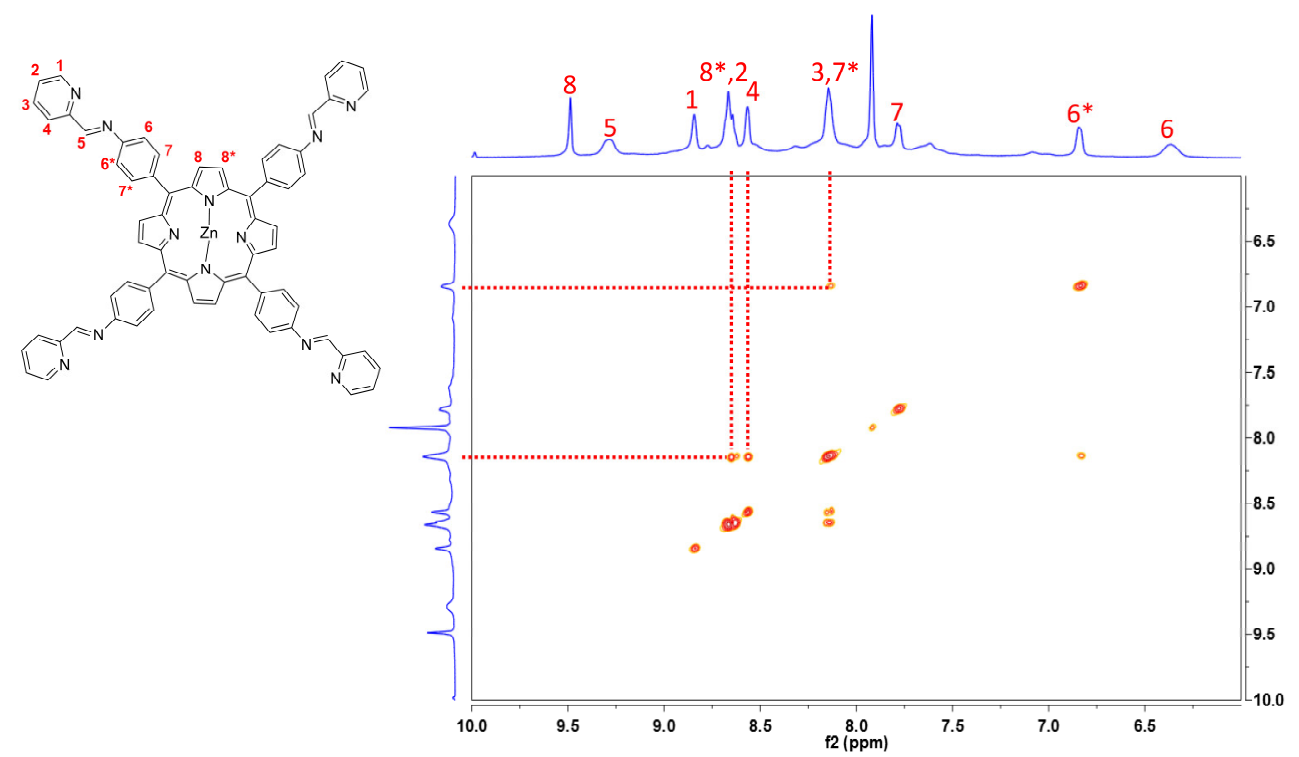

Figure S13. ${ }^{1} \mathrm{H}-{ }^{1} \mathrm{H}$ COSY spectrum $(500 \mathrm{M}, 298 \mathrm{~K})$ for $\mathbf{Z n - 1}$ in $\mathrm{CD}_{3} \mathrm{CN}:$ DMF- $d^{7}=3: 2$.

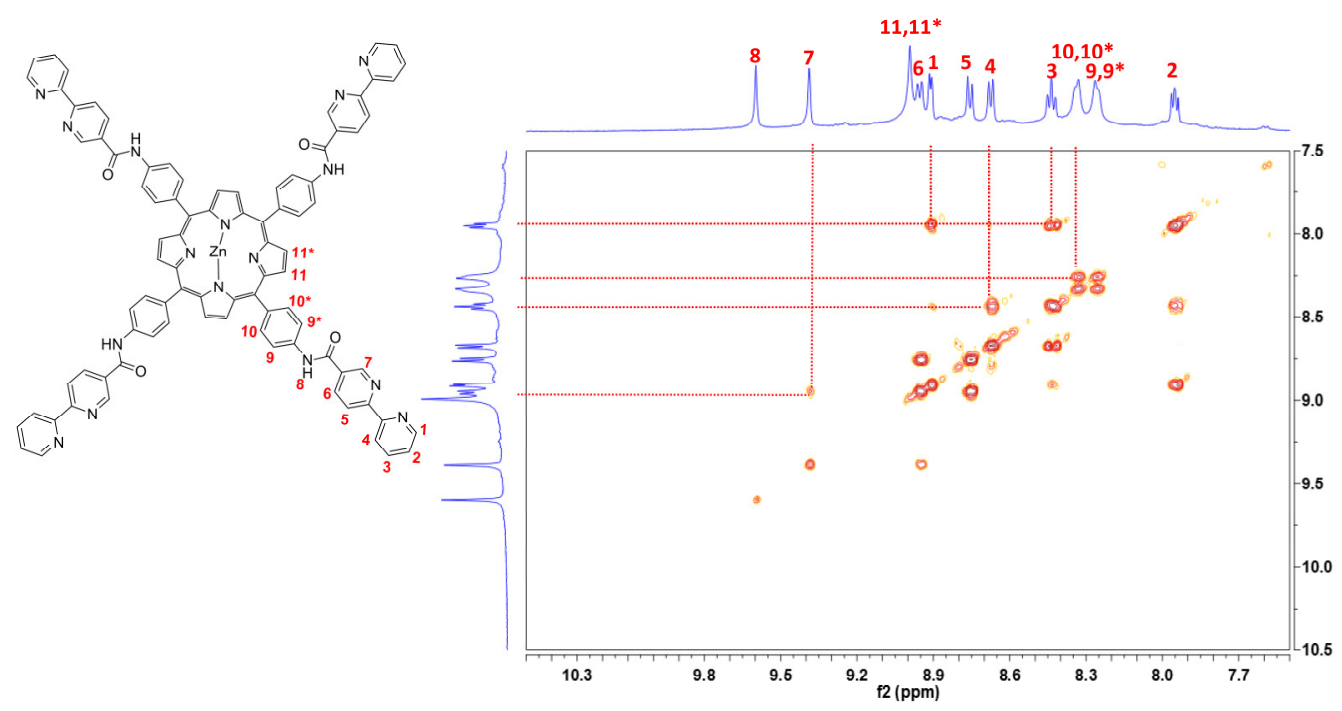

Figure S14. ${ }^{1} \mathrm{H}-{ }^{1} \mathrm{H}$ COSY spectrum $(500 \mathrm{M}, 298 \mathrm{~K})$ for $\mathbf{Z n - 2}$ in $\mathrm{CD}_{3} \mathrm{CN}$.

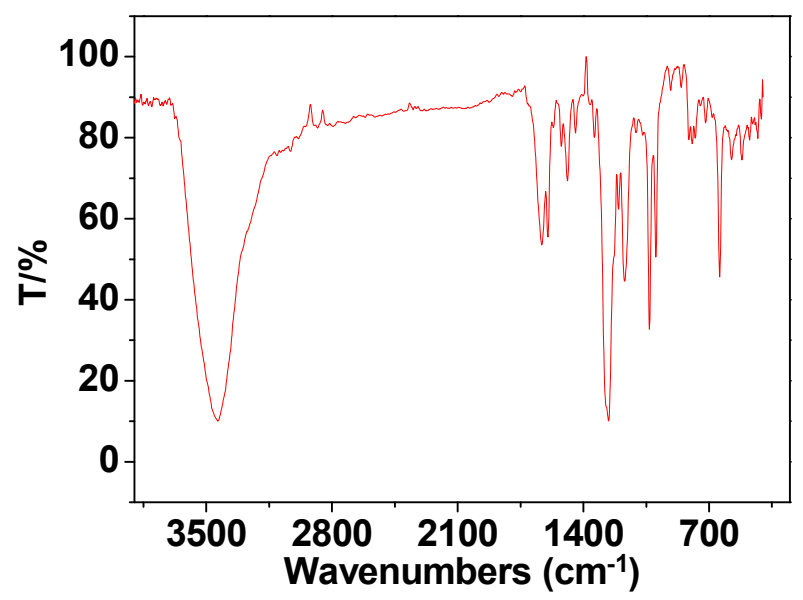


Figure S15. IR spectra for Zn-1.

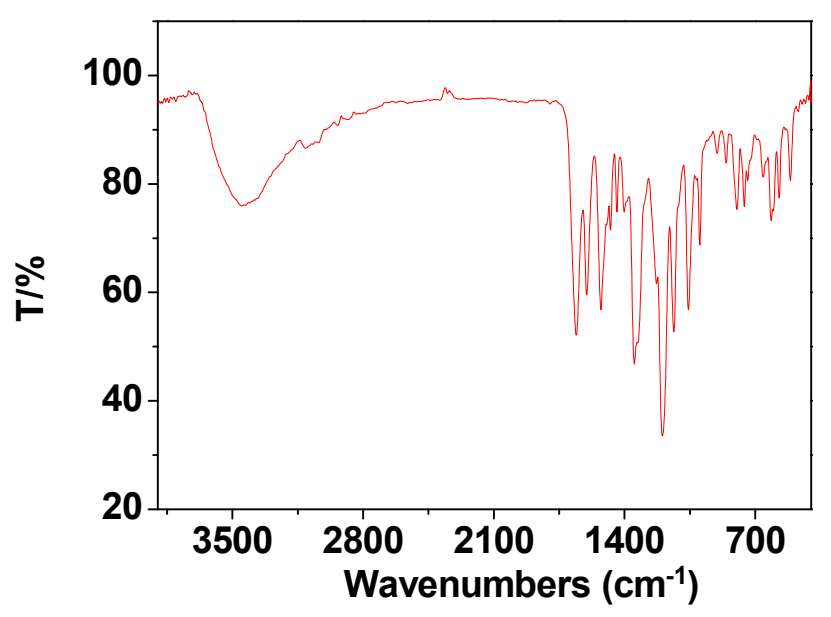

Figure S16. IR spectra for $\mathbf{Z n - 2}$.

Table S1. Crystal data and structure refinement for Zn-1.

\begin{tabular}{cc}
\hline Identification code & $\mathbf{Z n - 1}$ \\
\hline Formula & $\mathrm{C}_{474} \mathrm{H}_{394} \mathrm{~F}_{48} \mathrm{~N}_{78} \mathrm{O}_{66} \mathrm{~S}_{16} \mathrm{Zn}_{14}$ \\
Formula weight & 10578.8 \\
$\mathrm{~T} / \mathrm{K}$ & $200(2)$ \\
Crystal system & Tetragonal \\
Space group & $P 4 / n$ \\
Cell parameters & $\mathrm{a}=34.401(5) \AA, \mathrm{b}=34.401(5) \AA, \mathrm{c}=$ \\
& $27.321(4) \AA, \beta=90^{\circ}$ \\
Volume $\left(\AA^{3}\right), \mathrm{Z}$ & $32333(8)$ \\
$D_{c}\left(\mathrm{~g} \mathrm{~cm}{ }^{-3}\right)$ & 1.087 \\
$\left.\mu(\mathrm{mm})^{-1}\right)$ & 0.633 \\
$\mathrm{~F}(000)$ & 10840 \\
$R_{\text {int }}$ & $28190 / 5632 / 2924$ \\
$\mathrm{R}_{1}[\mathrm{I}>2 \sigma(\mathrm{I})]$ & 0.0566 \\
$w R_{2}($ all data $)$ & 0.0997 \\
Goodness of Fit & 0.2624 \\
$\mathrm{CCDC}$ No. & 0.976 \\
\hline
\end{tabular}

\section{Explanations of $\mathbf{A} / \mathbf{B}$ alerts:}

The A alert in checkcif was caused by the poor quality of the crystal resulting weak diffraction intensities. Most of the B alerts in checkcif were caused by the presence of highly disordered coordinated and free solvent molecules, some atoms in these moieties 
have higher thermal parameters. And the solvent water molecules could not be located well. And the highly disordered state of the incorporated molecule solvents meant that lots of them could not be located, and hence in the final refinement, the electron density was treated with the SQUEEZE routine in the PLATON program package. To assist the refinements, DAMP was used and some disagreeable reflections were omitted.

Table S2. Selected bond lengths $[\AA]$ and angles $\left[^{\circ}\right]$ for $\mathbf{Z n - 1}$.

\begin{tabular}{|c|c|c|c|c|}
\hline $\mathrm{Zn}(1)-\mathrm{N}\left(13^{\prime}\right)$ & \multicolumn{2}{|c|}{$2.013(6)$} & $\mathrm{Zn}(4)-\mathrm{N}(1)$ & $2.043(6)$ \\
\hline $\mathrm{Zn}(1)-\mathrm{N}\left(15^{\prime}\right)$ & \multicolumn{2}{|c|}{$2.087(8)$} & $\mathrm{Zn}(4)-\mathrm{N}(2)$ & $2.069(6)$ \\
\hline $\mathrm{Zn}(1)-\mathrm{N}(16) \# 1$ & \multicolumn{2}{|c|}{$2.144(4)$} & $\mathrm{Zn}(4)-\mathrm{N}(3)$ & $2.099(7)$ \\
\hline $\mathrm{Zn}(1)-\mathrm{N}(15)$ & \multicolumn{2}{|c|}{$2.152(6)$} & $\mathrm{Zn}(4)-\mathrm{O}(400)$ & $2.135(7)$ \\
\hline $\mathrm{Zn}(1)-\mathrm{N}(11)$ & \multicolumn{2}{|c|}{$2.190(4)$} & $\mathrm{Zn}(4)-\mathrm{N}(4)$ & $2.146(5)$ \\
\hline $\mathrm{Zn}(1)-\mathrm{N}(14)$ & \multicolumn{2}{|c|}{$2.190(4)$} & $\mathrm{Zn}(2)-\mathrm{N}(23)$ & $2.074(4)$ \\
\hline $\mathrm{Zn}(1)-\mathrm{N}(12) \# 1$ & \multicolumn{2}{|c|}{$2.205(4)$} & $\mathrm{Zn}(2)-\mathrm{N}(23) \# 1$ & $2.075(4)$ \\
\hline $\mathrm{Zn}(1)-\mathrm{N}(13)$ & \multicolumn{2}{|c|}{$2.313(10)$} & $\mathrm{Zn}(2)-\mathrm{N}(23) \# 2$ & $2.075(4)$ \\
\hline $\mathrm{Zn}(3)-\mathrm{N}\left(10^{\prime}\right)$ & \multicolumn{2}{|c|}{$2.012(10)$} & $\mathrm{Zn}(2)-\mathrm{N}(23) \# 3$ & $2.075(4)$ \\
\hline $\mathrm{Zn}(3)-\mathrm{N}(8)$ & \multicolumn{2}{|c|}{$2.080(5)$} & $\mathrm{Zn}(2)-\mathrm{O}(1 \mathrm{~W})$ & $2.126(10)$ \\
\hline $\mathrm{Zn}(3)-\mathrm{N}(9) \# 2$ & \multicolumn{2}{|c|}{$2.127(11)$} & $\mathrm{Zn}(5)-\mathrm{N}(17)$ & $2.048(5)$ \\
\hline $\mathrm{Zn}(3)-\mathrm{N}(5) \# 2$ & \multicolumn{2}{|c|}{$2.171(4)$} & $\mathrm{Zn}(5)-\mathrm{N}(17) \# 3$ & $2.048(5)$ \\
\hline $\mathrm{Zn}(3)-\mathrm{N}(10)$ & \multicolumn{2}{|c|}{$2.173(11)$} & $\mathrm{Zn}(5)-\mathrm{N}(17) \# 2$ & $2.048(5)$ \\
\hline $\mathrm{Zn}(3)-\mathrm{N}(7)$ & \multicolumn{2}{|c|}{$2.175(4)$} & $\mathrm{Zn}(5)-\mathrm{N}(17) \# 1$ & $2.048(5)$ \\
\hline $\mathrm{Zn}(3)-\mathrm{N}(6)$ & \multicolumn{2}{|c|}{$2.198(4)$} & $\mathrm{Zn}(5)-\mathrm{O}(3 \mathrm{~W})$ & $2.209(12)$ \\
\hline $\mathrm{Zn}(3)-\mathrm{N}\left(8^{\prime}\right)$ & \multicolumn{2}{|c|}{$2.405(13)$} & $\mathrm{Zn}\left(5^{\prime}\right)-\mathrm{N}\left(17^{\prime}\right) \# 2$ & 2.094(11) \\
\hline $\mathrm{Zn}\left(2^{\prime}\right)-\mathrm{N}(23)$ & \multicolumn{2}{|c|}{$2.065(4)$} & $\mathrm{Zn}\left(5^{\prime}\right)-\mathrm{N}\left(17^{\prime}\right)$ & $2.094(11)$ \\
\hline $\mathrm{Zn}\left(2^{\prime}\right)-\mathrm{N}(23) \# 2$ & \multicolumn{2}{|c|}{$2.065(4)$} & $\mathrm{Zn}\left(5^{\prime}\right)-\mathrm{O}(4 \mathrm{~W})$ & $2.084(19)$ \\
\hline $\mathrm{Zn}\left(2^{\prime}\right)-\mathrm{N}(23) \# 1$ & \multicolumn{2}{|c|}{$2.065(4)$} & $\mathrm{Zn}\left(4^{\prime}\right)-\mathrm{N}\left(2^{\prime}\right)$ & $2.042(10)$ \\
\hline $\mathrm{Zn}\left(2^{\prime}\right)-\mathrm{N}(23) \# 3$ & \multicolumn{2}{|c|}{$2.065(4)$} & $\mathrm{Zn}\left(4^{\prime}\right)-\mathrm{N}\left(4^{\prime}\right)$ & $2.057(10)$ \\
\hline $\mathrm{Zn}\left(2^{\prime}\right)-\mathrm{O}(2 \mathrm{~W})$ & \multicolumn{2}{|c|}{$2.26(2)$} & $\mathrm{Zn}\left(4^{\prime}\right)-\mathrm{N}\left(3^{\prime}\right)$ & $2.061(10)$ \\
\hline $\mathrm{Zn}\left(5^{\prime}\right)-\mathrm{N}\left(17^{\prime}\right) \# 3$ & \multicolumn{2}{|c|}{$2.094(11)$} & $\mathrm{Zn}\left(4^{\prime}\right)-\mathrm{N}\left(1^{\prime}\right)$ & $2.190(8)$ \\
\hline $\mathrm{Zn}\left(5^{\prime}\right)-\mathrm{N}\left(17^{\prime}\right) \# 1$ & \multicolumn{2}{|c|}{$2.094(11)$} & $\mathrm{Zn}\left(4^{\prime}\right)-\mathrm{O}(850)$ & $2.189(13)$ \\
\hline \multicolumn{3}{|c|}{$\mathrm{N}\left(13^{\prime}\right)-\mathrm{Zn}(1)-\mathrm{N}(16) \# 1$} & $\mathrm{~N}(5) \# 2-\mathrm{Zn}(3)-\mathrm{N}(7)$ & $95.60(15)$ \\
\hline \multicolumn{2}{|c|}{$\mathrm{N}\left(15^{\prime}\right)-\mathrm{Zn}(1)-\mathrm{N}(16) \# 1$} & $93.9(3)$ & $\mathrm{N}(10)-\mathrm{Zn}(3)-\mathrm{N}(7)$ & $165.0(3)$ \\
\hline \multicolumn{2}{|c|}{ N(13')-Zn(1)-N(15) } & $93.7(3)$ & $\mathrm{N}\left(10^{\prime}\right)-\mathrm{Zn}(3)-\mathrm{N}(6)$ & $76.5(3)$ \\
\hline \multicolumn{2}{|c|}{$\mathrm{N}(16) \# 1-\mathrm{Zn}(1)-\mathrm{N}(15)$} & $101.5(2)$ & $\mathrm{N}(8)-\mathrm{Zn}(3)-\mathrm{N}(6)$ & $92.6(2)$ \\
\hline \multicolumn{2}{|c|}{$\mathrm{N}\left(13^{\prime}\right)-\mathrm{Zn}(1)-\mathrm{N}(11)$} & $76.9(2)$ & $\mathrm{N}(9) \# 2-\mathrm{Zn}(3)-\mathrm{N}(6)$ & $170.3(3)$ \\
\hline \multicolumn{2}{|c|}{$\mathrm{N}\left(15^{\prime}\right)-\mathrm{Zn}(1)-\mathrm{N}(11)$} & $173.5(3)$ & $\mathrm{N}(5) \# 2-\mathrm{Zn}(3)-\mathrm{N}(6)$ & $94.79(15)$ \\
\hline \multicolumn{2}{|c|}{$\mathrm{N}(16) \# 1-\mathrm{Zn}(1)-\mathrm{N}(11)$} & $92.21(16)$ & $\mathrm{N}(10)-\mathrm{Zn}(3)-\mathrm{N}(6)$ & $75.3(3)$ \\
\hline $\mathrm{N}(15)-\mathrm{Zn}(1)-\mathrm{N}(1$ & & $164.2(2)$ & $\mathrm{N}(7)-\mathrm{Zn}(3)-\mathrm{N}(6)$ & $92.01(16)$ \\
\hline $\mathrm{N}\left(13^{\prime}\right)-\mathrm{Zn}(1)-\mathrm{N}($ & & $94.0(3)$ & $\mathrm{N}\left(10^{\prime}\right)-\mathrm{Zn}(3)-\mathrm{N}\left(8^{\prime}\right)$ & $98.1(5)$ \\
\hline $\mathrm{N}\left(15^{\prime}\right)-\mathrm{Zn}(1)-\mathrm{N}($ & & $81.6(3)$ & $\mathrm{N}(5) \# 2-\mathrm{Zn}(3)-\mathrm{N}\left(8^{\prime}\right)$ & $165.7(4)$ \\
\hline
\end{tabular}




\begin{tabular}{llll}
\hline $\mathrm{N}(16) \# 1-\mathrm{Zn}(1)-\mathrm{N}(14)$ & $169.18(15)$ & $\mathrm{N}(7)-\mathrm{Zn}(3)-\mathrm{N}\left(8^{\prime}\right)$ & $73.2(3)$ \\
$\mathrm{N}(15)-\mathrm{Zn}(1)-\mathrm{N}(14)$ & $75.9(2)$ & $\mathrm{N}(6)-\mathrm{Zn}(3)-\mathrm{N}\left(8^{\prime}\right)$ & $94.4(5)$ \\
$\mathrm{N}(11)-\mathrm{Zn}(1)-\mathrm{N}(14)$ & $91.99(15)$ & $\mathrm{N}(1)-\mathrm{Zn}(4)-\mathrm{N}(2)$ & $91.3(2)$ \\
$\mathrm{N}\left(13^{\prime}\right)-\mathrm{Zn}(1)-\mathrm{N}(12) \# 1$ & $168.8(2)$ & $\mathrm{N}(1)-\mathrm{Zn}(4)-\mathrm{N}(3)$ & $87.8(2)$ \\
$\mathrm{N}\left(15^{\prime}\right)-\mathrm{Zn}(1)-\mathrm{N}(12) \# 1$ & $85.1(3)$ & $\mathrm{N}(2)-\mathrm{Zn}(4)-\mathrm{N}(3)$ & $161.3(3)$ \\
$\mathrm{N}(16) \# 1-\mathrm{Zn}(1)-\mathrm{N}(12) \# 1$ & $76.46(16)$ & $\mathrm{N}\left(100^{\prime}\right)-\mathrm{Zn}(3)-\mathrm{N}(5) \# 2$ & $94.7(4)$ \\
$\mathrm{N}(15)-\mathrm{Zn}(1)-\mathrm{N}(12) \# 1$ & $96.3(2)$ & $\mathrm{N}(8)-\mathrm{Zn}(3)-\mathrm{N}(5) \# 2$ & $170.4(2)$ \\
$\mathrm{N}(11)-\mathrm{Zn}(1)-\mathrm{N}(12) \# 1$ & $94.37(14)$ & $\mathrm{N}(9) \# 2-\mathrm{Zn}(3)-\mathrm{N}(5) \# 2$ & $77.6(3)$ \\
$\mathrm{N}(14)-\mathrm{Zn}(1)-\mathrm{N}(12) \# 1$ & $93.28(14)$ & $\mathrm{N}(8)-\mathrm{Zn}(3)-\mathrm{N}(10)$ & $94.3(4)$ \\
$\mathrm{N}\left(15^{\prime}\right)-\mathrm{Zn}(1)-\mathrm{N}(13)$ & $104.3(4)$ & $\mathrm{N}(9) \# 2-\mathrm{Zn}(3)-\mathrm{N}(10)$ & $99.0(4)$ \\
$\mathrm{N}(16) \# 1-\mathrm{Zn}(1)-\mathrm{N}(13)$ & $100.9(4)$ & $\mathrm{N}(5) \# 2-\mathrm{Zn}(3)-\mathrm{N}(10)$ & $93.5(3)$ \\
$\mathrm{N}(11)-\mathrm{Zn}(1)-\mathrm{N}(13)$ & $76.5(3)$ & $\mathrm{N}(10)-\mathrm{Zn}(3)-\mathrm{N}(7)$ & $165.2(3)$ \\
$\mathrm{N}(14)-\mathrm{Zn}(1)-\mathrm{N}(13)$ & $89.8(4)$ & $\mathrm{N}(8)-\mathrm{Zn}(3)-\mathrm{N}(7)$ & $78.1(2)$ \\
$\mathrm{N}(12) \# 1-\mathrm{Zn}(1)-\mathrm{N}(13)$ & $170.4(3)$ & $\mathrm{N}(9) \# 2-\mathrm{Zn}(3)-\mathrm{N}(7)$ & $94.6(4)$ \\
$\mathrm{N}(8)-Z n(3)-\mathrm{N}(9) \# 2$ & $95.6(3)$ & &
\end{tabular}

Symmetry transformations used to generate equivalent atoms:

$\# 1 \mathrm{y}-1,-\mathrm{x}+3 / 2, \mathrm{z} \quad \# 2-\mathrm{y}+3 / 2, \mathrm{x}+1, \mathrm{z} \quad \# 3-\mathrm{x}+1 / 2,-\mathrm{y}+5 / 2, \mathrm{z}$

\subsection{Synthesis of the substrates.}

\subsubsection{Synthesis of (5-Phenyl-pyridin-2-yl)-methanol (1a)}

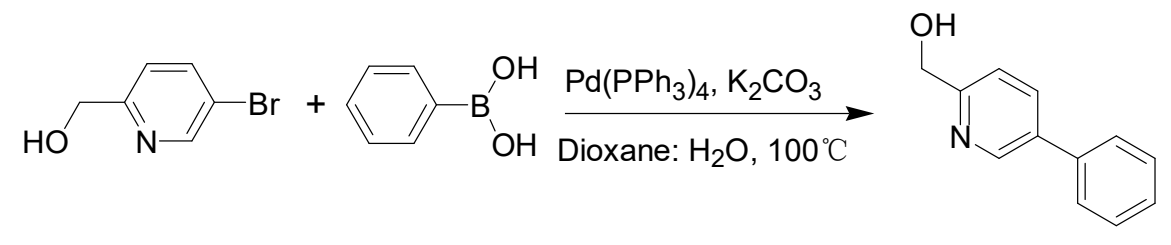

A mixture of 2-hydroxymethyl-5-bromopyridine $(0.41 \mathrm{~g}, 2.2 \mathrm{mmol})$, phenylboronic acid (0.24 g, $2 \mathrm{mmol}), \mathrm{K}_{3} \mathrm{PO}_{4}(0.3 \mathrm{~g}, 2.2 \mathrm{mmol})$ and $\mathrm{Pd}\left(\mathrm{PPh}_{3}\right)_{4}(210 \mathrm{mg}, 0.2 \mathrm{mmol})$ in dioxane $/ \mathrm{H}_{2} \mathrm{O}(3: 1 \mathrm{v} / \mathrm{v}, 40 \mathrm{~mL})$ was degassed for three times and the suspension was stirred under $\mathrm{Ar}$ at $100{ }^{\circ} \mathrm{C}$ for $72 \mathrm{~h}$. After cooling to r.t., the mixture was concentrated and $\mathrm{CH}_{2} \mathrm{Cl}_{2}$ (200mL) was added. The combined organic phase was washed with brine, dried over $\mathrm{Na}_{2} \mathrm{SO}_{4}$ and then concentrated under reduced pressure. The crude product was purified by column chromatography on silica gel $\left(\mathrm{CH}_{2} \mathrm{Cl}_{2} /\right.$ EtOAc, $\left.1: 1 \mathrm{v} / \mathrm{v}\right)$ to afford (5-phenyl-pyridin-2-yl)-methanol (1a) as a white solid (yield 70\%).

${ }^{1}$ H NMR (400 MHz, CDCl 3$): \delta=8.69(1 \mathrm{H}, \mathrm{d}, \mathrm{J}=1.6 \mathrm{~Hz}), 7.80(1 \mathrm{H}, \mathrm{dd}, \mathrm{J}=4 \mathrm{~Hz}, 8 \mathrm{~Hz})$, $7.50(2 \mathrm{H}, \mathrm{d}, \mathrm{J}=8 \mathrm{~Hz}), 7.41(2 \mathrm{H}, \mathrm{t}, \mathrm{J}=8 \mathrm{~Hz}), 7.33(1 \mathrm{H}, \mathrm{d}, \mathrm{J}=8 \mathrm{~Hz}), 7.28(1 \mathrm{H}, \mathrm{d}, \mathrm{J}=8 \mathrm{~Hz})$, $4.74(2 \mathrm{H}, \mathrm{s}), 4.06(1 \mathrm{H}, \mathrm{s})$. 
${ }^{13}$ C NMR (400 MHz, $\left.\mathbf{C D C l}_{3}\right): \delta=157.10,145.95,136.50,134.22,128.07,127.06$, 126.04, 119.55, 63.20.

\subsubsection{Synthesis of [5-(4-Methoxy-phenyl)-pyridin-2-yl]-methanol (1b)}

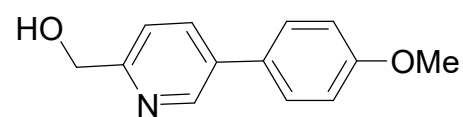

Prepared by following the experimental procedure in section 2.2.1 from 4methoxyphenylboronic acid $(0.3 \mathrm{~g}, 2 \mathrm{mmol})$ and purified by flash column chromatography $\left(\mathrm{CH}_{2} \mathrm{Cl}_{2} /\right.$ EtOAc, $\left.1: 1 \mathrm{v} / \mathrm{v}\right)$ to give $\mathbf{1 b}$ in $75 \%$ yield.

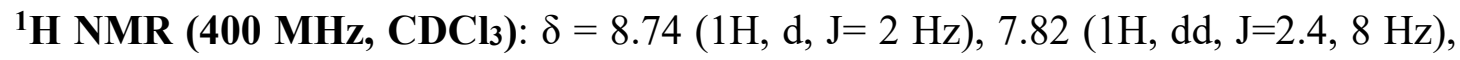
7.50-7.52 (2H, m), $7.30(1 \mathrm{H}, \mathrm{d}, \mathrm{J}=8 \mathrm{~Hz}), 7.01-7.02(2 \mathrm{H}, \mathrm{m}), 4.80(2 \mathrm{H}, \mathrm{s}), 3.86(3 \mathrm{H}, \mathrm{s})$. ${ }^{13}$ C NMR (400 MHz, $\left.\mathbf{C D C l}_{3}\right): \delta=170.77,159.86,153.73,147.67,135.64,134.63$, $128.24,122.00,114.61,66.82,20.97$.

\subsubsection{Synthesis of (5-Biphenyl-4-yl-pyridin-2-yl)-methanol (1c)}

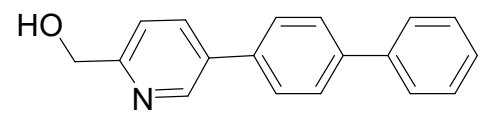

Prepared by following the experimental procedure in section 2.2.1 from 4biphenylboronic acid ( $0.4 \mathrm{~g}, 2 \mathrm{mmol})$ and purified by flash column chromatography $\left(\mathrm{CH}_{2} \mathrm{Cl}_{2} / \mathrm{EtOAc}, 1: 1 \mathrm{v} / \mathrm{v}\right)$ to give $1 \mathrm{c}$ in $80 \%$ yield.

${ }^{1}$ H NMR (400 MHz, DMSO- $d^{6}$ ): $\delta=8.86(1 \mathrm{H}, 2 \mathrm{~Hz}), 8.15(1 \mathrm{H}, \mathrm{dd}, \mathrm{J}=2.4,8 \mathrm{~Hz}), 7.82$ (4H, q, J=8, $14.4 \mathrm{~Hz}), 7.74(2 \mathrm{H}, \mathrm{d}, 8 \mathrm{~Hz}), 7.57(1 \mathrm{H}, \mathrm{d}, \mathrm{J}=8 \mathrm{~Hz}), 7.50(2 \mathrm{H}, \mathrm{t}, \mathrm{J}=8 \mathrm{~Hz})$, $7.40(1 \mathrm{H}, \mathrm{t}, \mathrm{J}=7.2 \mathrm{~Hz}), 5.54(1 \mathrm{H}, \mathrm{s}), 4.64(2 \mathrm{H}, \mathrm{s})$.

${ }^{13}$ C NMR (400 MHz, DMSO-d $\left.d^{6}\right): \delta=160.98,146.37,139.62,139.44,136.08,134.43$, $133.16,128.98,127.61,127.32,127.20,126.58,120.27,64.08$.

\subsubsection{Synthesis of (5-(anthracen-9-yl)pyridin-2-yl) methanol (1d)}

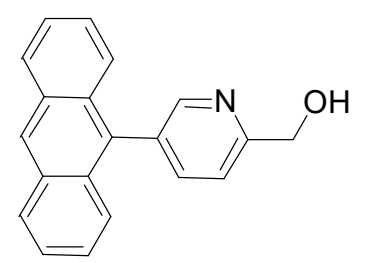

Prepared by following the experimental procedure in section 2.2.1 from 4biphenylboronic acid $(0.4 \mathrm{~g}, 2 \mathrm{mmol})$ and purified by flash column chromatography 
$\left(\mathrm{CH}_{2} \mathrm{Cl}_{2} /\right.$ EtOAc, $\left.3: 1 \mathrm{v} / \mathrm{v}\right)$ to give $\mathbf{1 d}$ in $74 \%$ yield.

${ }^{1} \mathbf{H}$ NMR (400 MHz, CDCl 3$): \delta=8.06(2 \mathrm{H}, \mathrm{d}, \mathrm{J}=8 \mathrm{~Hz}), 7.78(1 \mathrm{H}, \mathrm{s}, \mathrm{J}=4 \mathrm{~Hz}), 7.60(2$ H, d, J=8 Hz), $7.50(3 \mathrm{H}, \mathrm{q}, \mathrm{J}=8,16 \mathrm{~Hz}), 7.39(2 \mathrm{H}, \mathrm{t}, \mathrm{J}=8 \mathrm{~Hz}), 4.96(2 \mathrm{H}, \mathrm{s}), 3.78(1 \mathrm{H}, \mathrm{s})$. ${ }^{13}$ C NMR (400 MHz, $\left.\mathbf{C D C l}_{3}\right): \delta=158.23,150.46,146.63,139.61,137.98$, $135.52,133.32,132.31,130.56,128.59,128.29,127.89,127.62,126.01,125.30,123.82$, $122.88,120.21,120.18,64.29$.

\subsubsection{Preparation (6-phenylpyridin-2-y) methanol}

\subsubsection{Synthesis of $\mathbf{N}, \mathbf{N}$-dimethyl-6-phenylpiconlinamide}

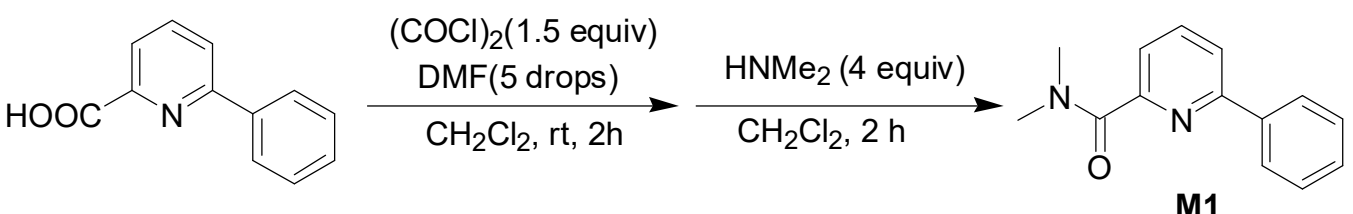

To a solution of 6-phenylpyridine-2-carboxylic acid (1.0 g, $5 \mathrm{mmol})$ in $\mathrm{CH}_{2} \mathrm{Cl}_{2}(20 \mathrm{~mL})$ was added $(\mathrm{COCl})_{2}(0.65 \mathrm{~mL}, 7.5 \mathrm{mmol})$ and $\mathrm{DMF}(5$ drops $)$ at $23{ }^{\circ} \mathrm{C}$. The reaction mixture was stirred at room temperature for $2 \mathrm{~h}$, before adding $\mathrm{Me}_{2} \mathrm{NH}$ (40 wt\% in water; $2.5 \mathrm{~mL}, 20 \mathrm{mmol}$ ) dropwise at $0{ }^{\circ} \mathrm{C}$, and the reaction mixture was stirred continuously at $23{ }^{\circ} \mathrm{C}$ for $2 \mathrm{~h}$. the reaction was then quenched with water and organic materials were extracted twice with $\mathrm{CH}_{2} \mathrm{Cl}_{2}$, washed with $1 \mathrm{M}$ aqueous $\mathrm{HCl}$ solution followed by saturated aqueous $\mathrm{NaHCO}_{3}$ solution and brine, and then dried over $\mathrm{MgSO}_{4}$. The solvent was removed in vacuo and the resulting crude mixture was purified by column chromatography ( silica gel, hexane: ethyl acetate $=20: 80)$ to give M1 $(960 \mathrm{mg})$ in $84.7 \%$ yield.

${ }^{1} \mathbf{H}$ NMR (400 MHz, CDCl 3$): \delta=8.03-8.00(2 \mathrm{H}, \mathrm{m}), 7.87-7.83(1 \mathrm{H}, \mathrm{m}), 7.76(1 \mathrm{H}, \mathrm{dd}$, $\mathrm{J}=1.2,8.0 \mathrm{~Hz}), 7.60(1 \mathrm{H}, \mathrm{dd}, \mathrm{J}=1.2,7.2 \mathrm{~Hz}), 7.50-7.41(3 \mathrm{H}, \mathrm{m}), 3.20(3 \mathrm{H}, \mathrm{s}), 3.18(3 \mathrm{H}$, s).

\subsubsection{Synthesis of (6-phenylpyridin-2-y)methanol (1g)}

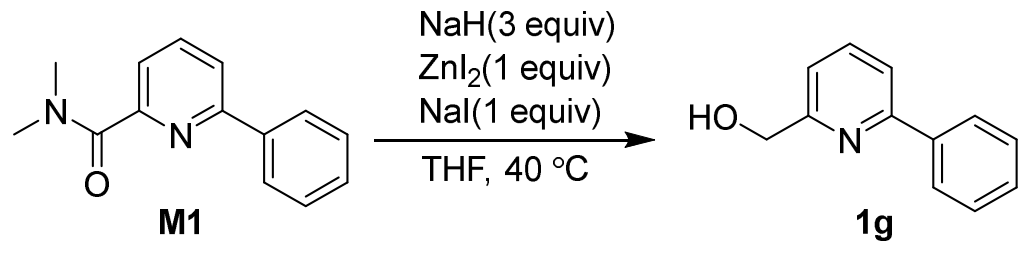


To a mixture of $\mathrm{NaH}$ (60\% dispersion in mineral oil; $0.480 \mathrm{~g}, 12 \mathrm{mmol}), \mathrm{NaI}(0.6 \mathrm{~g}, 4$ $\mathrm{mmol})$ and $\mathrm{ZnI}_{2}(1.28 \mathrm{~g}, 4.0 \mathrm{mmol})$ in a $50 \mathrm{~mL}$ 2-neck round-bottom flask was added a solution of M1 (0.90 g, $4.0 \mathrm{mmol})$ in $20 \mathrm{~mL}$ of THF. The reaction mixture was placed under $\mathrm{N}_{2}$ atmosphere and stirred at $40{ }^{\circ} \mathrm{C}$ for $7 \mathrm{~h}$. The reaction was quenched with ammonium $\mathrm{pH} 10$ buffer at $0{ }^{\circ} \mathrm{C}$ and the organic materials were extracted with dichloromethane $(10 \mathrm{~mL} \times 3)$. The combined extracts were dried over $\mathrm{MgSO}_{4}$. The volatile materials were removed in vacuo and the resulting crude residue was purified by flash column chromatography ( silica gel, Hexane: EtOAc $=25: 1)$ to give $\mathbf{1 g}(0.62$ g) in 84\% yield. ${ }^{1} \mathbf{H}$ NMR (400 MHz, $\left.\mathbf{C D C l}_{3}\right): \delta=8.0(2 \mathrm{H}, \mathrm{d}, \mathrm{J}=8 \mathrm{~Hz}), 7.74(1 \mathrm{H}, \mathrm{t}, \mathrm{J}=6$ Hz), $7.64(1 \mathrm{H}, \mathrm{d}, \mathrm{J}=4 \mathrm{~Hz}), 7.48(2 \mathrm{H}, \mathrm{t}, \mathrm{J}=6 \mathrm{~Hz}), 7.42(1 \mathrm{H}, \mathrm{t}, \mathrm{J}=6 \mathrm{~Hz}), 4.81$ (2H, s), 4.15 $(1 \mathrm{H}, \mathrm{s})$.

\subsubsection{Preparation 1-(1H-Imidazol-1-yl)-3-methyl-1-butanone (2e)}

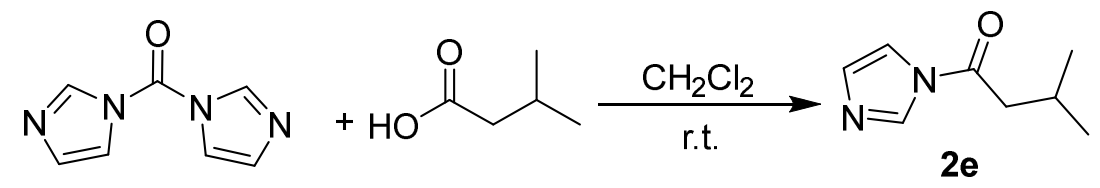

3-Methylbutyric acid (0.44 mL, $4 \mathrm{mmol})$ was dissolved in dry dichloromethane (15 mL) and $\mathrm{N}, \mathrm{N}$-carbonyldiimidazole $(0.97 \mathrm{~g}, 6 \mathrm{mmol})$ was added at $0{ }^{\circ} \mathrm{C}$. The resulting mixture was stirred for $2 \mathrm{~h}$ at r.t., washed with $\mathrm{CH}_{2} \mathrm{Cl}_{2}$ and saturated $\mathrm{NaHCO}_{3}$. After drying over $\mathrm{MgSO}_{4}$, residue solvent was removed under reduced pressure to give the colorless oil 2e (0.414 g) in 68\% yield. ${ }^{1} \mathbf{H}$ NMR (400 MHz, $\left.\mathbf{C D C l}_{3}\right): \delta=8.16(1 \mathrm{H}, \mathrm{s})$, $7.48(1 \mathrm{H}, \mathrm{t}, \mathrm{J}=1.6 \mathrm{~Hz}), 7.10(1 \mathrm{H}, \mathrm{s}), 2.74(2 \mathrm{H}, \mathrm{d}, \mathrm{J}=4 \mathrm{~Hz}), 2.32(1 \mathrm{H}, \mathrm{m}), 1.05$ (6H, d, $\mathrm{J}=8 \mathrm{~Hz}) .{ }^{13} \mathbf{C}$ NMR (400 MHz, $\left.\mathbf{C D C l}_{3}\right): \delta=168.84,136.16,130.98,116.03,43.98$, 25.30, 22.43 . 


\subsection{UV-Vis spectroscopy.}

Host-guest model: The experimental data obtained using UV-Vis spectroscopy for each guest was fitted using nonlinear analysis with DynaFit program (Biokin Software $)^{6}$ to the binding equation derived for 1:1 model porphyrin: imidazole/pyridine group (non-cooperative model). This model is ideal for the small substrate, such as NAI, 2-PC, 3-PC, 4-PC, imidazole and so on, which is so small that the binding to one porphyrin does not affect the interaction with the rest of porphyrins present in $\mathbf{Z n - 1}$ or Zn-2. Therefore, each porphyrin of cage must bind to one substrate molecule in the saturation point, with the six affinity constants having the same value. The decrease in absorption was also observed in the cases of the other guests. As shown in Figure S17S46, the behavior observed was similar for these guests. We thus inferred that in these cases each porphyrin unit of $\mathbf{Z n - 1}$ or $\mathbf{Z n - 2}$ was interacting with one imidazole/pyridine group in the saturation point of the titration. In this way, a non-cooperative model was used to obtain the intrinsic dissociation constant for the all of the guests.
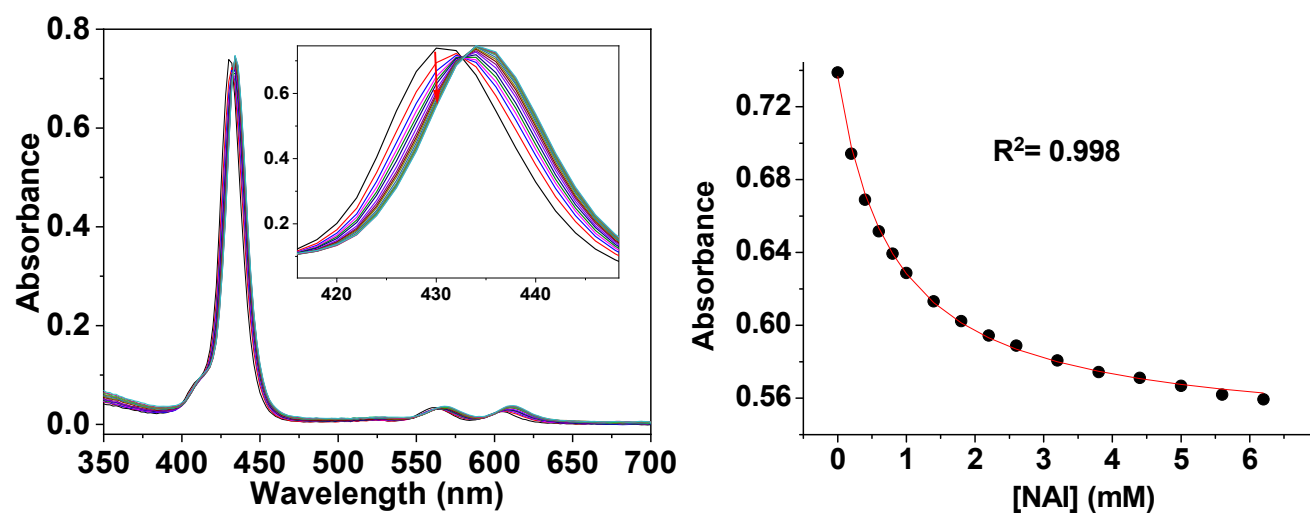

Figure S17. UV-Vis titration of NAI in $\mathrm{CH}_{3} \mathrm{CN}$ : DMF (3:1 in volume). (a) Titration of $\mathbf{Z n - 1}(0.25$ $\mu \mathrm{M}$ ) with NAI as monitored by UV-Vis. (b) Binding isotherm from UV-Vis analysis at $430 \mathrm{~nm}$. $\left(K_{a}=1224 \pm 50 \mathrm{M}^{-1}\right.$ at $\left.25^{\circ} \mathrm{C}\right)$. 

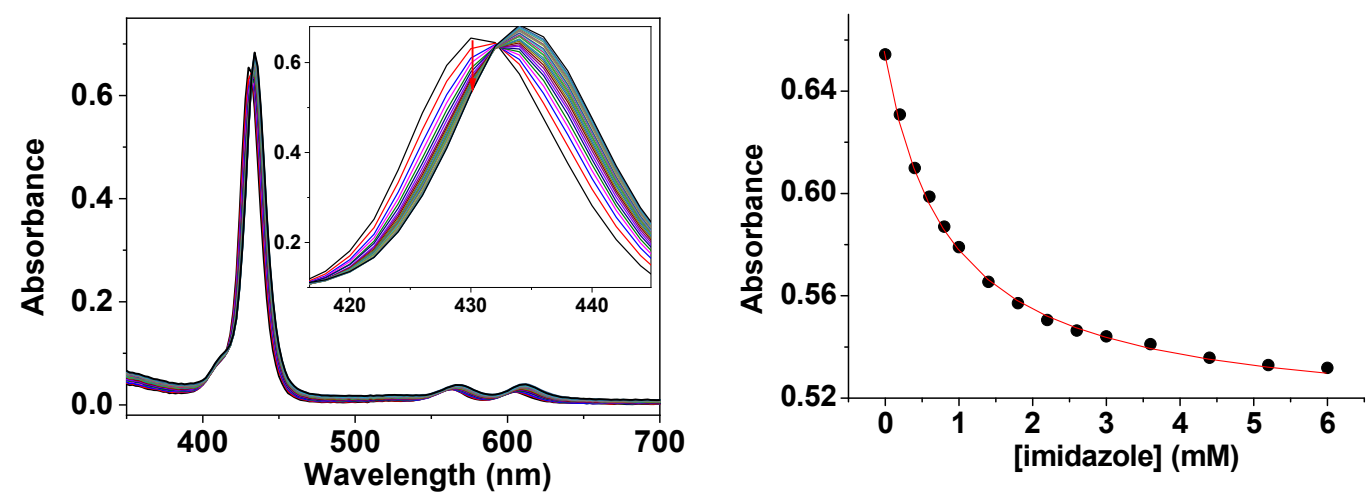

Figure S18. UV-Vis titration of imidazole in $\mathrm{CH}_{3} \mathrm{CN}$ : DMF (3:1 in volume). (a) Titration of $\mathbf{Z n - 1}$ $(0.25 \mu \mathrm{M})$ with imidazole as monitored by UV-Vis. (b) Binding isotherm from UV-Vis analysis at $430 \mathrm{~nm}\left(K_{a}=1160 \pm 47 \mathrm{M}^{-1}\right.$ at $\left.25^{\circ} \mathrm{C}\right)$.
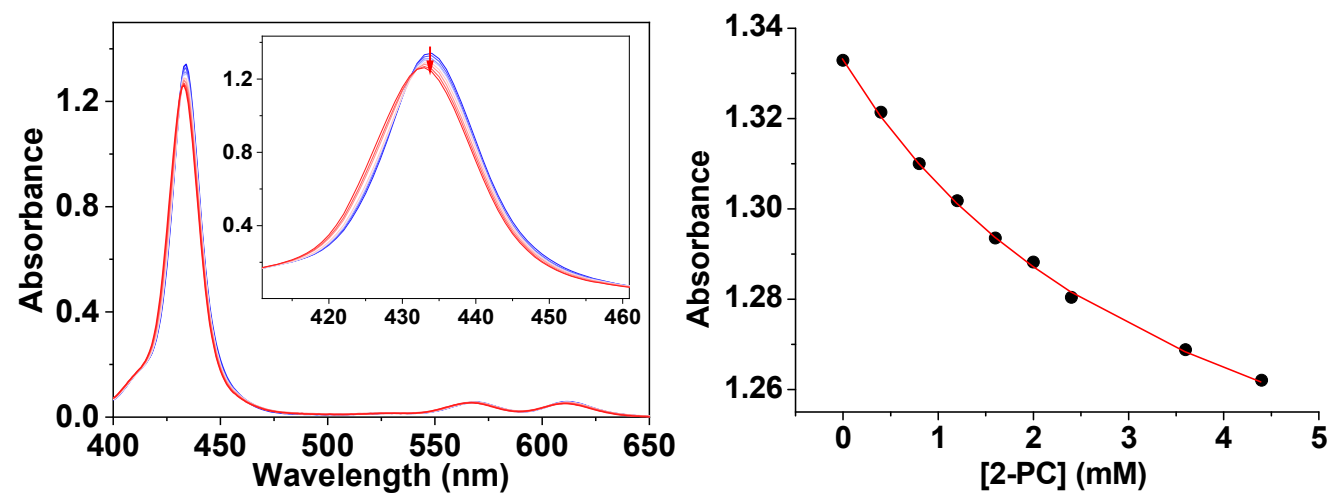

Figure S19. UV-Vis titration of 2-PC in $\mathrm{CH}_{3} \mathrm{CN}$ : DMF (3:1 in volume). (a) Titration of $\mathbf{Z n - 1}(0.5$ $\mu \mathrm{M}$ ) with 2-PC as monitored by UV-Vis. (b) Binding isotherm from UV-Vis analysis at $430 \mathrm{~nm}$ $\left(K_{a}=262 \pm 19 \mathrm{M}^{-1}\right.$ at $\left.25^{\circ} \mathrm{C}\right)$.
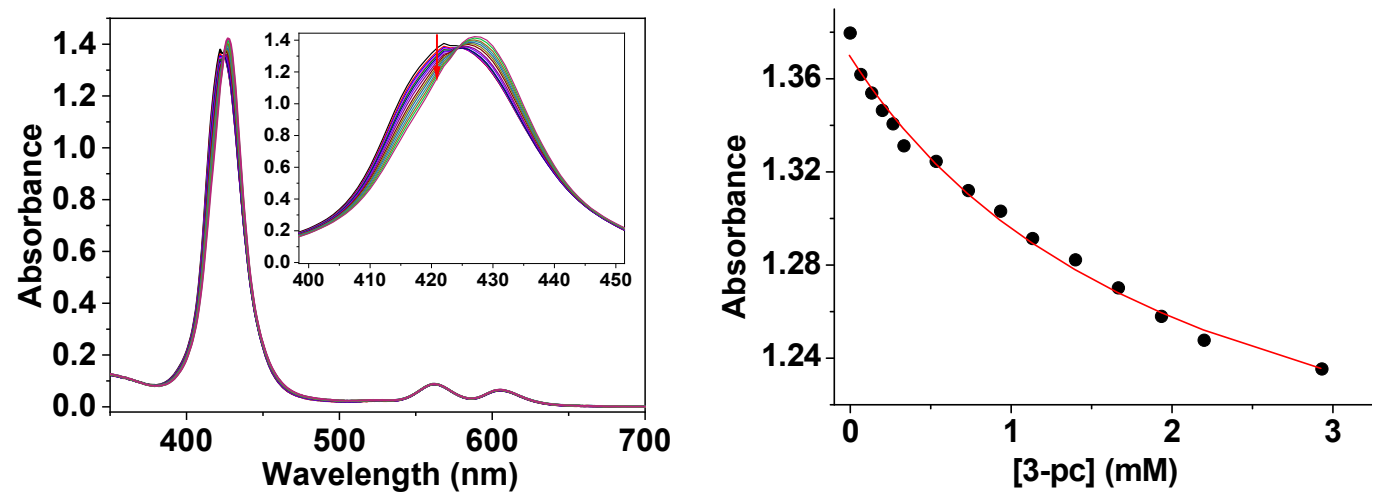

Figure S20. UV-Vis titration of 3-PC in $\mathrm{CH}_{3} \mathrm{CN}$ : DMF (3:1 in volume). (a) Titration of $\mathbf{Z n - 1}$ (0.5 $\mu \mathrm{M}$ ) with 3-PC as monitored by UV-Vis. (b) Binding isotherm from UV-Vis analysis at $430 \mathrm{~nm}$ $\left(K_{a}=467 \pm 87 \mathrm{M}^{-1}\right.$ at $\left.25^{\circ} \mathrm{C}\right)$. 

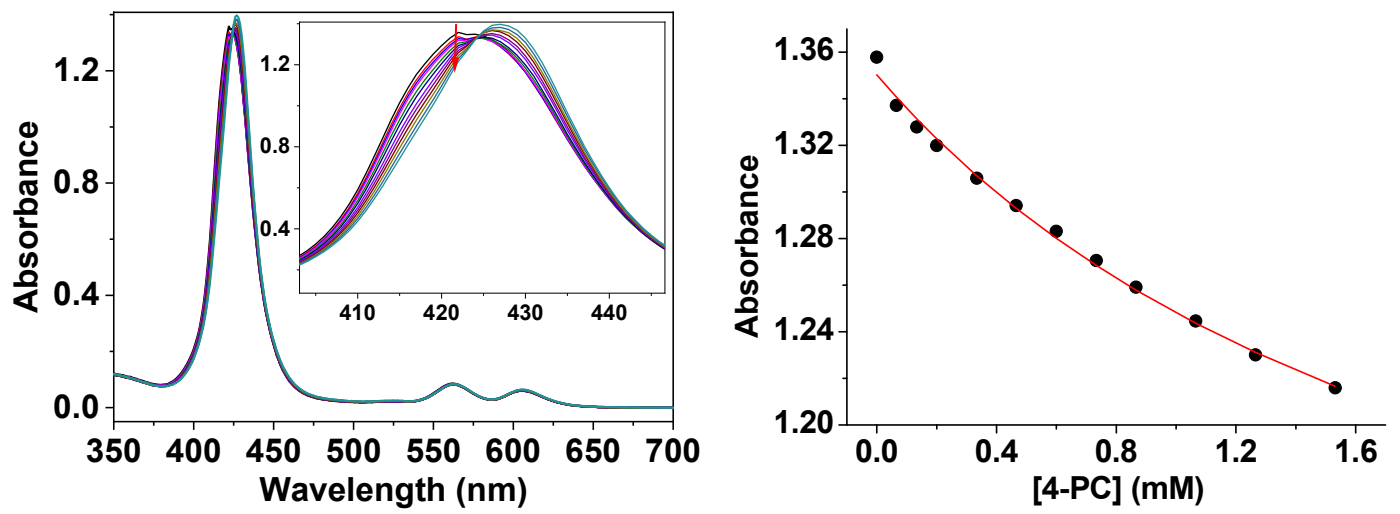

Figure S21. UV-Vis titration of 4-PC in $\mathrm{CH}_{3} \mathrm{CN}$ : DMF (3:1 in volume). (a) Titration of Zn-1 (0.5 $\mu \mathrm{M})$ with 4-PC as monitored by UV-Vis. (b) Binding isotherm from UV-Vis analysis at $430 \mathrm{~nm}\left(K_{a}=\right.$ $470 \pm 94 \mathrm{M}^{-1}$ at $\left.25^{\circ} \mathrm{C}\right)$.

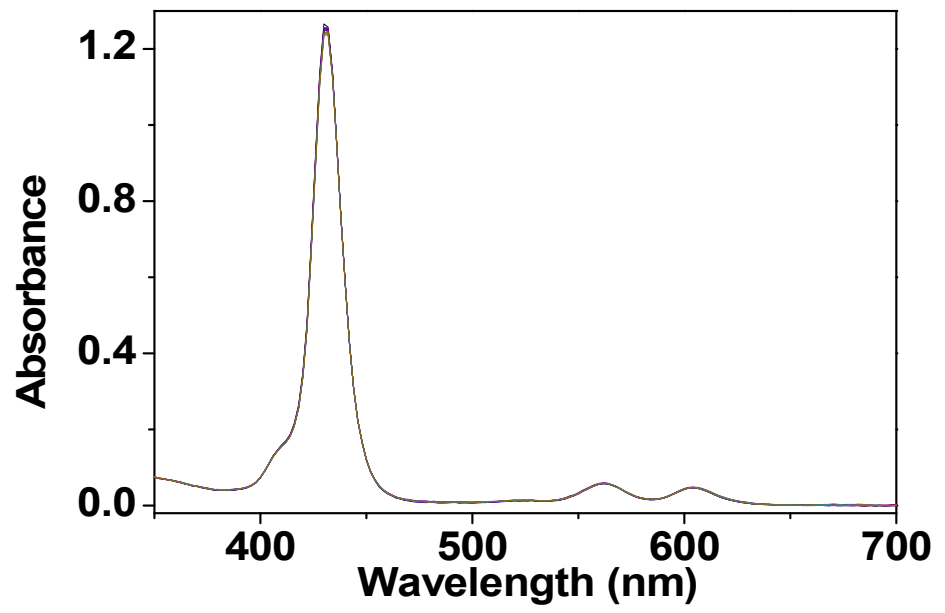

Figure S22. Titration of $\mathbf{Z n - 1}$ with 2-AMPy $(0.5 \mu \mathrm{M})$ in $\mathrm{CH}_{3} \mathrm{CN}$ : DMF (3:1 in volume) as monitored by UV-Vis $\left(25^{\circ} \mathrm{C}\right)$.
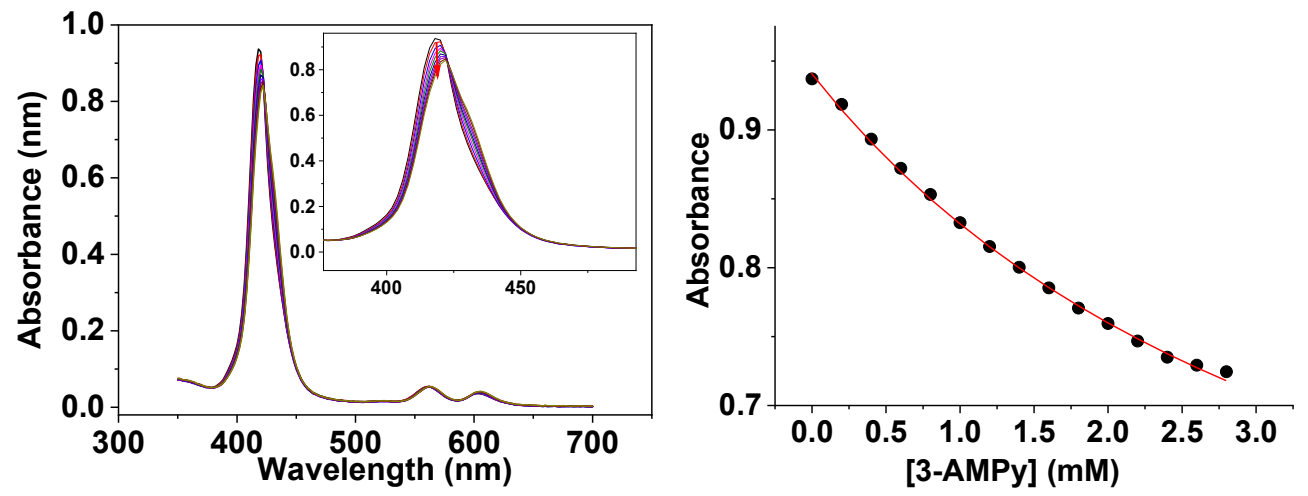

Figure S23. UV-Vis titration of 3-AMPy in $\mathrm{CH}_{3} \mathrm{CN}$ : DMF (3:1 in volume). (a) Titration of $\mathbf{Z n - 1}$ $(0.5 \mu \mathrm{M})$ with 3-AMPy as monitored by UV-Vis. (b) Binding isotherm from UV-Vis analysis at $430 \mathrm{~nm}\left(K_{a}=256 \pm 24 \mathrm{M}^{-1}\right.$ at $\left.25^{\circ} \mathrm{C}\right)$. 

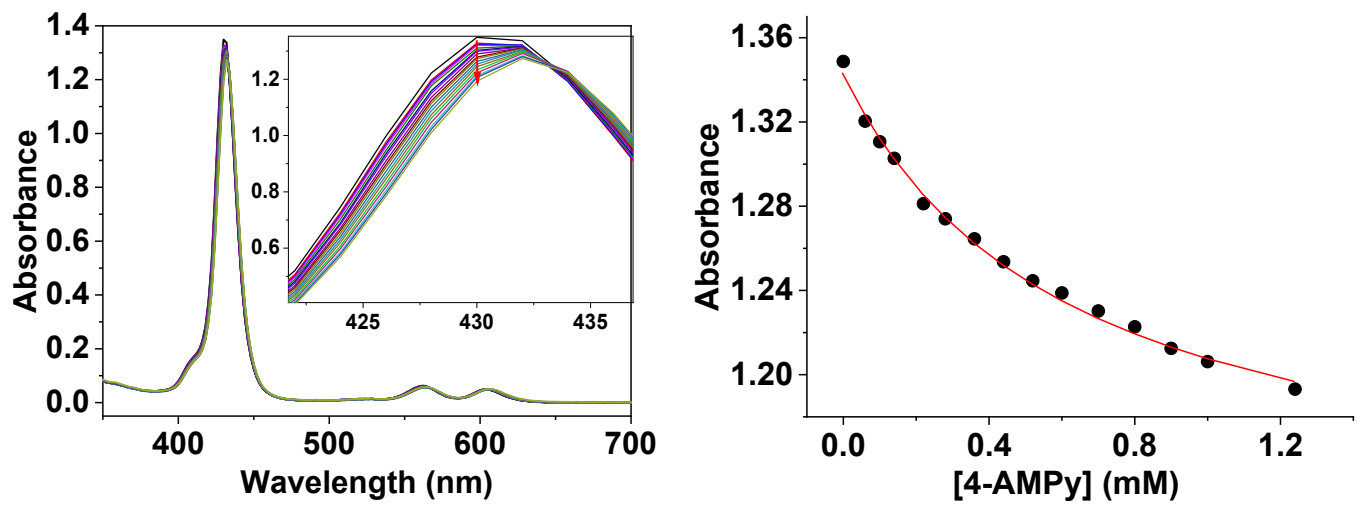

Figure S24. UV-Vis titration of 4-AMPy in $\mathrm{CH}_{3} \mathrm{CN}$ : DMF (3:1 in volume). (a) Titration of $\mathbf{Z n - 1}$ $(0.5 \mu \mathrm{M})$ with 4-AMPy as monitored by UV-Vis. (b) Binding isotherm from UV-Vis analysis at 430 $\mathrm{nm}\left(K_{a}=1510 \pm 151 \mathrm{M}^{-1}\right.$ at $\left.25^{\circ} \mathrm{C}\right)$.
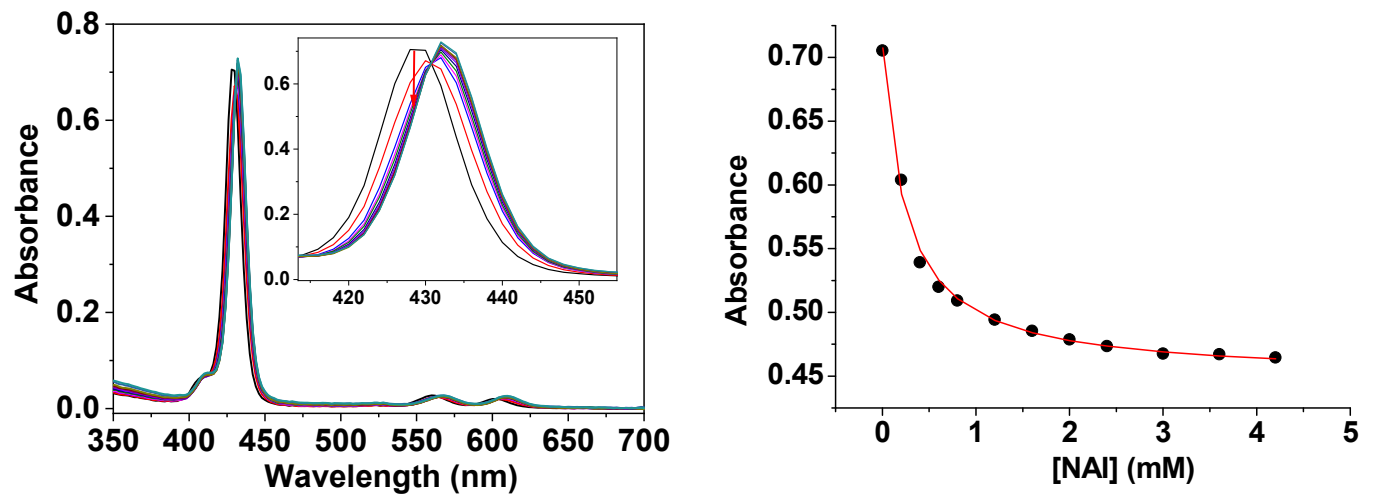

Figure S25. UV-Vis titration of NAI in $\mathrm{CH}_{3} \mathrm{CN}$ : DMF (3:1 in volume). (a) Titration of $\mathbf{Z n - 2}(0.25$ $\mu \mathrm{M})$ with NAI as monitored by UV-Vis. (b) Binding isotherm from UV-Vis analysis at $428 \mathrm{~nm}\left(K_{a}\right.$ $=4000 \pm 316 \mathrm{M}^{-1}$ at $25^{\circ} \mathrm{C}$ ).
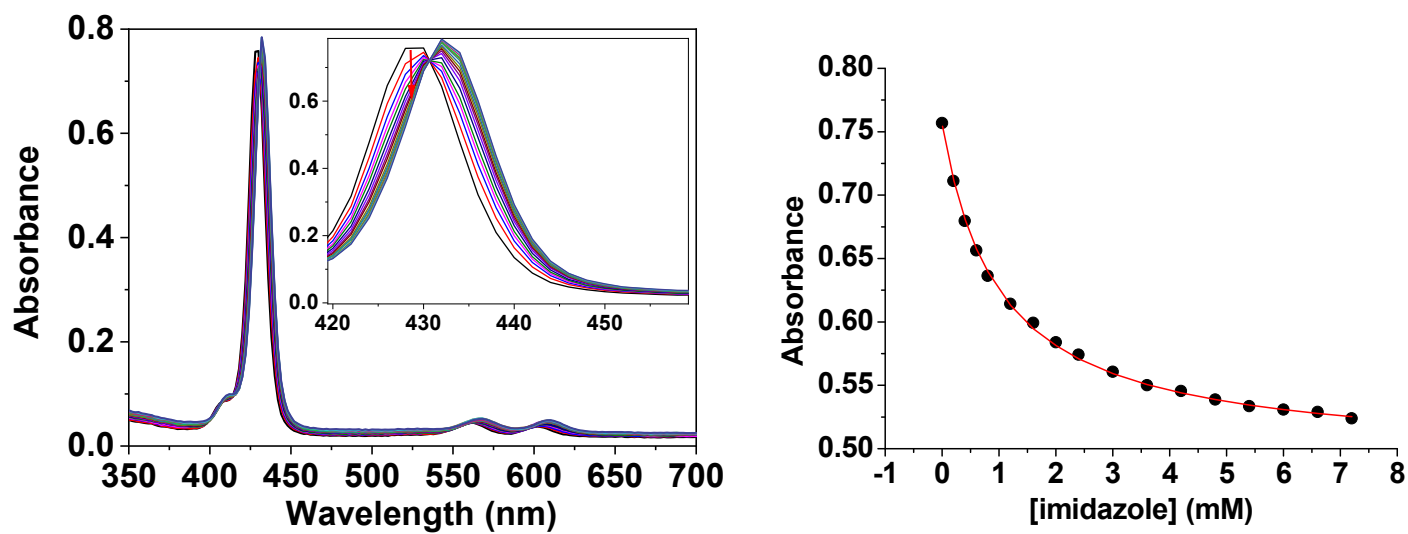

Figure S26. UV-Vis titration of imidazole in $\mathrm{CH}_{3} \mathrm{CN}$ : DMF (3:1 in volume). (a) Titration of $\mathbf{Z n - 2}$ $(0.25 \mu \mathrm{M})$ with imidazole as monitored by UV-Vis. (b) Binding isotherm from UV-Vis analysis at $428 \mathrm{~nm}\left(K_{a}=1000 \pm 31 \mathrm{M}^{-1}\right.$ at $\left.25^{\circ} \mathrm{C}\right)$. 

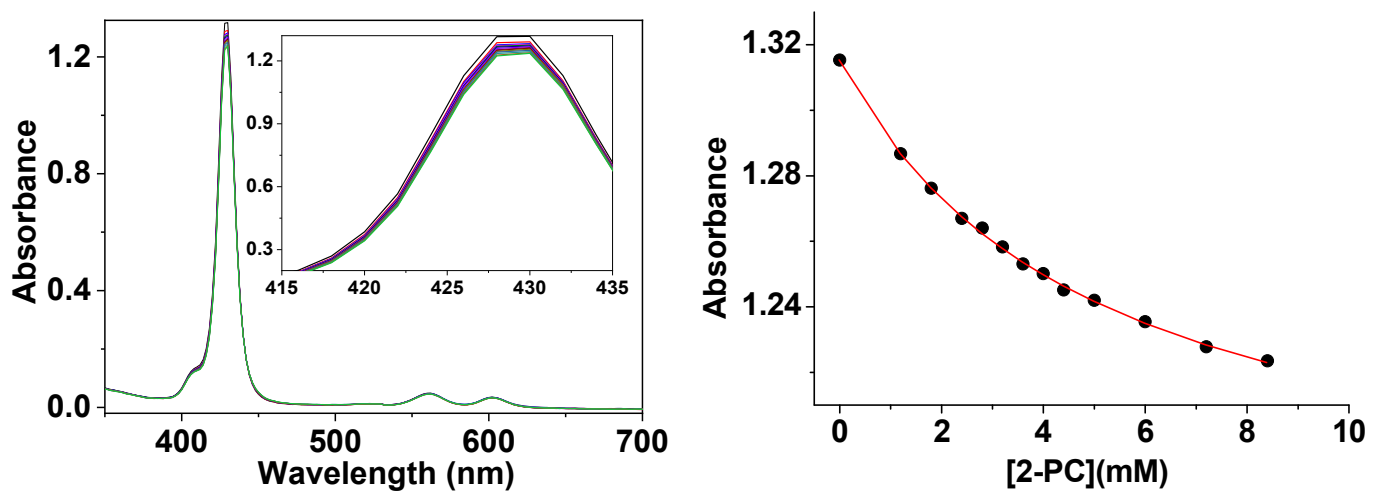

Figure S27. UV-Vis titration of 2-PC in $\mathrm{CH}_{3} \mathrm{CN}$ : DMF (3:1 in volume). (a) Titration of $\mathbf{Z n - 2}(0.5$ $\mu \mathrm{M})$ with 2-PC as monitored by UV-Vis. (b) Binding isotherm from UV-Vis analysis at $428 \mathrm{~nm}\left(K_{a}\right.$ $=200 \pm 8 \mathrm{M}^{-1}$ at $\left.25^{\circ} \mathrm{C}\right)$.
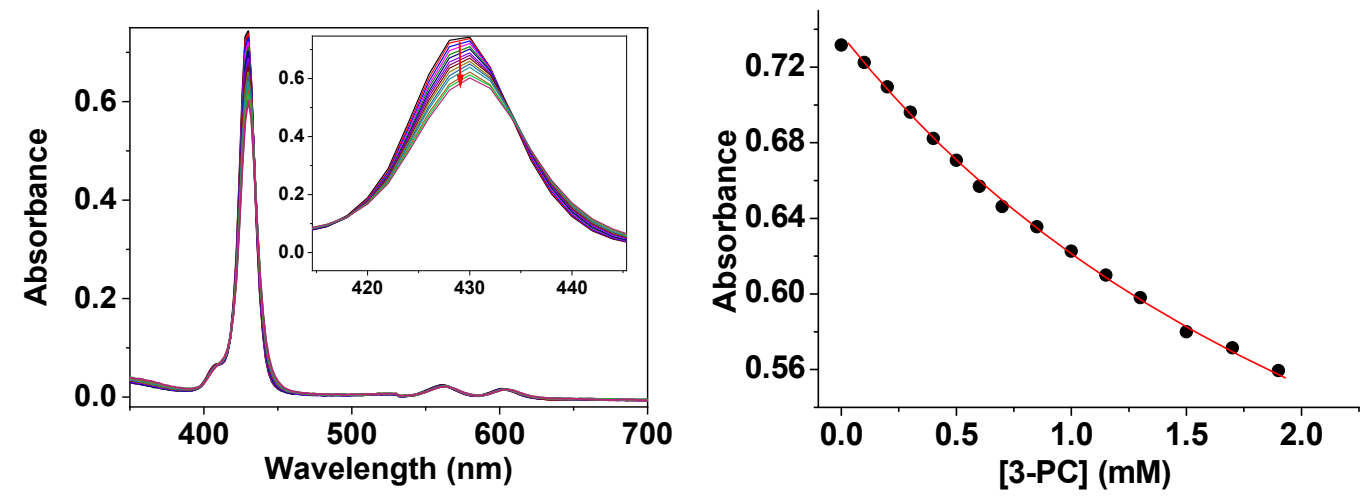

Figure S28. UV-Vis titration of 3-PC in $\mathrm{CH}_{3} \mathrm{CN}$ : DMF (3:1 in volume). (a) Titration of $\mathbf{Z n - 2}(0.25$ $\mu \mathrm{M})$ with 3-PC as monitored by UV-Vis. (b) Binding isotherm from UV-Vis analysis at $428 \mathrm{~nm}\left(K_{a}\right.$ $=333 \pm 33 \mathrm{M}^{-1}$ at $25^{\circ} \mathrm{C}$ ).
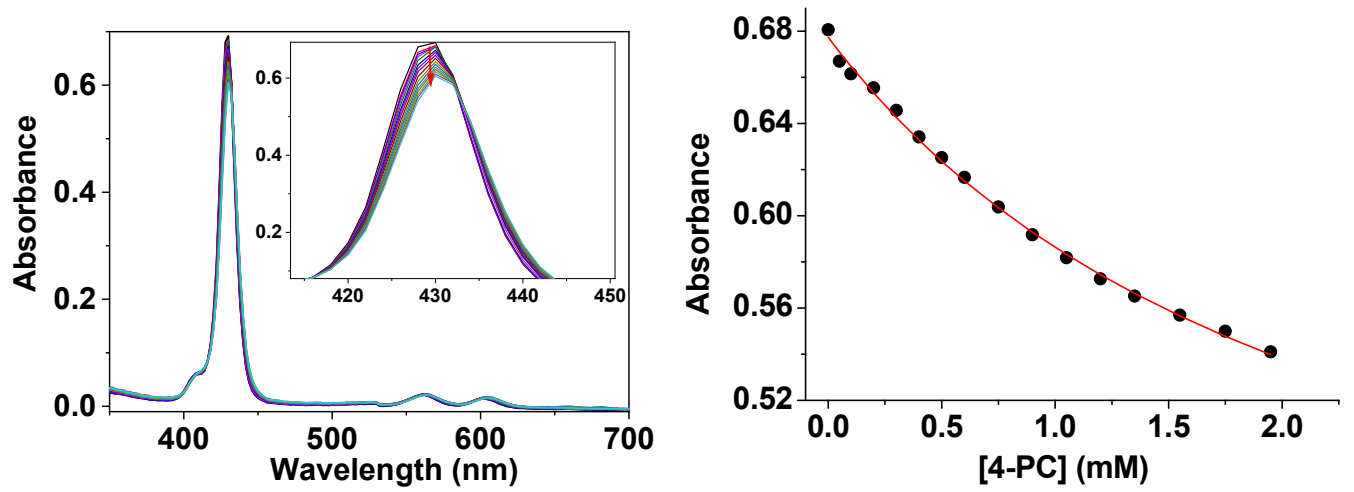

Figure S29. UV-Vis titration of 4-PC in $\mathrm{CH}_{3} \mathrm{CN}$ : DMF (3:1 in volume). (a) Titration of $\mathbf{Z n - 2}(0.25$ $\mu \mathrm{M})$ with 4-PC as monitored by UV-Vis. (b) Binding isotherm from UV-Vis analysis at $428 \mathrm{~nm}\left(K_{a}\right.$ $=438 \pm 46 \mathrm{M}^{-1}$ at $25^{\circ} \mathrm{C}$ ). 


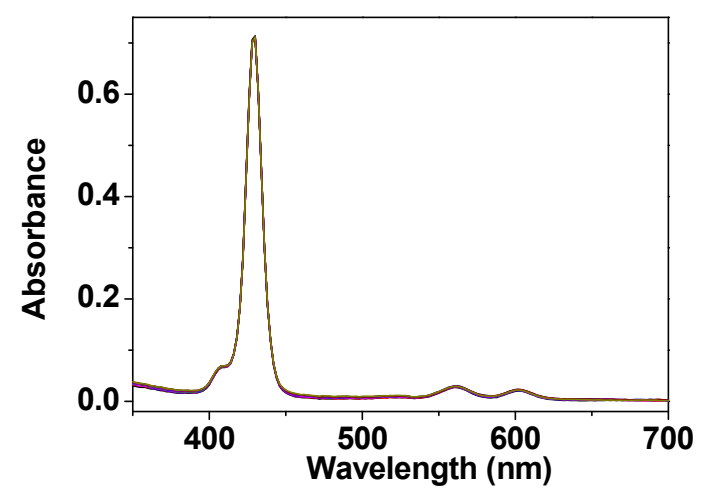

Figure S30. Titration of $\mathbf{Z n - 2}(0.25 \mu \mathrm{M})$ with 2-AMPy in $\mathrm{CH}_{3} \mathrm{CN}$ : DMF (3:1 in volume) as monitored by UV-Vis $\left(25^{\circ} \mathrm{C}\right)$.
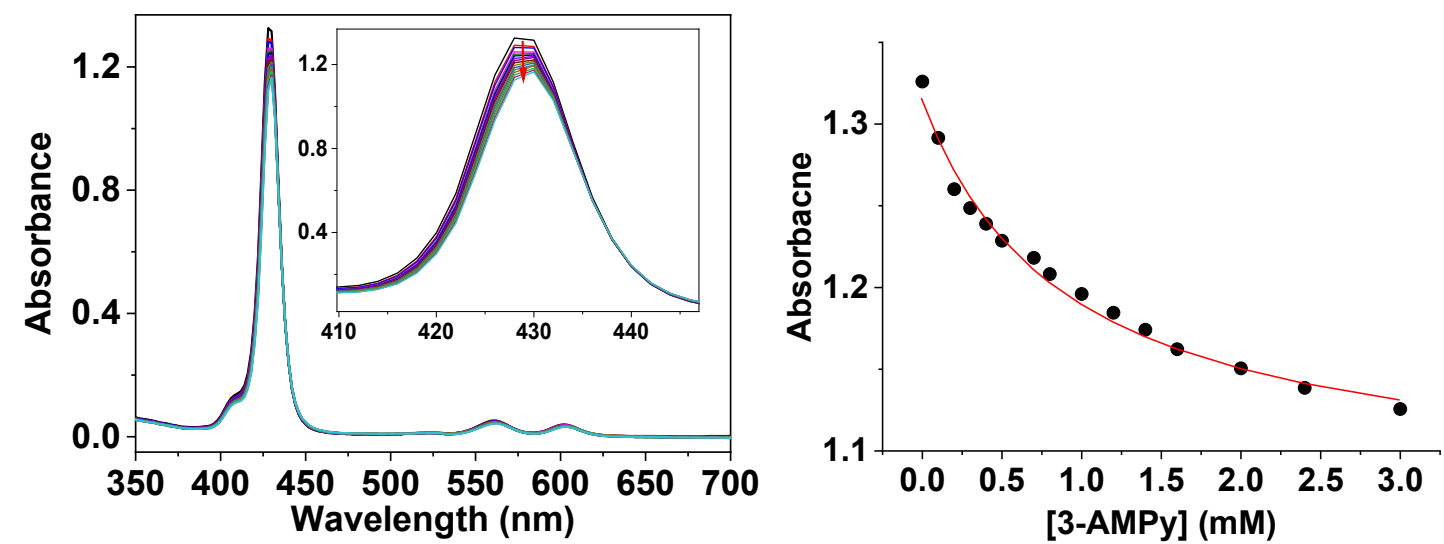

Figure S31. UV-Vis titration of 3-AMPy in $\mathrm{CH}_{3} \mathrm{CN}$ : DMF (3:1 in volume). (a) Titration of $\mathbf{Z n - 2}$ $(0.5 \mu \mathrm{M})$ with 3-AMPy as monitored by UV-Vis. (b) Binding isotherm from UV-Vis analysis at 428 $\mathrm{nm}\left(K_{a}=1111 \pm 162 \mathrm{M}^{-1}\right.$ at $\left.25^{\circ} \mathrm{C}\right)$.
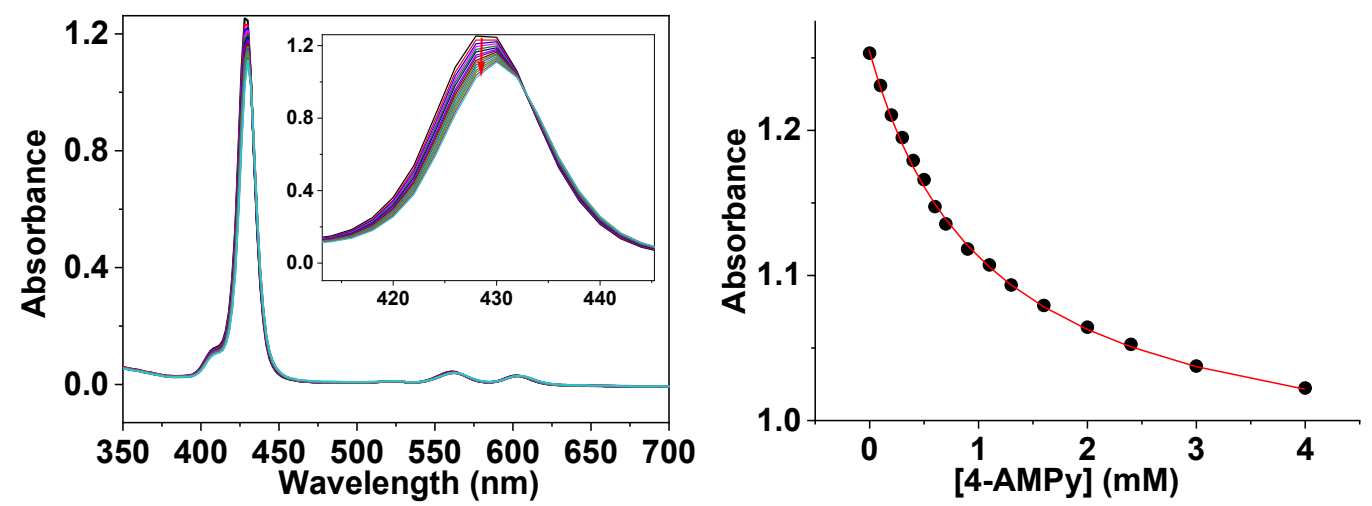

Figure S32. UV-Vis titration of 4-AMPy in $\mathrm{CH}_{3} \mathrm{CN}$ : DMF (3:1 in volume). (a) Titration of $\mathbf{Z n - 2}$ $(0.5 \mu \mathrm{M})$ with 4-AMPy as monitored by UV-Vis. (b) Binding isotherm from UV-Vis analysis at 428 $\mathrm{nm}\left(K_{a}=1000 \pm 41 \mathrm{M}^{-1}\right.$ at $\left.25^{\circ} \mathrm{C}\right)$. 


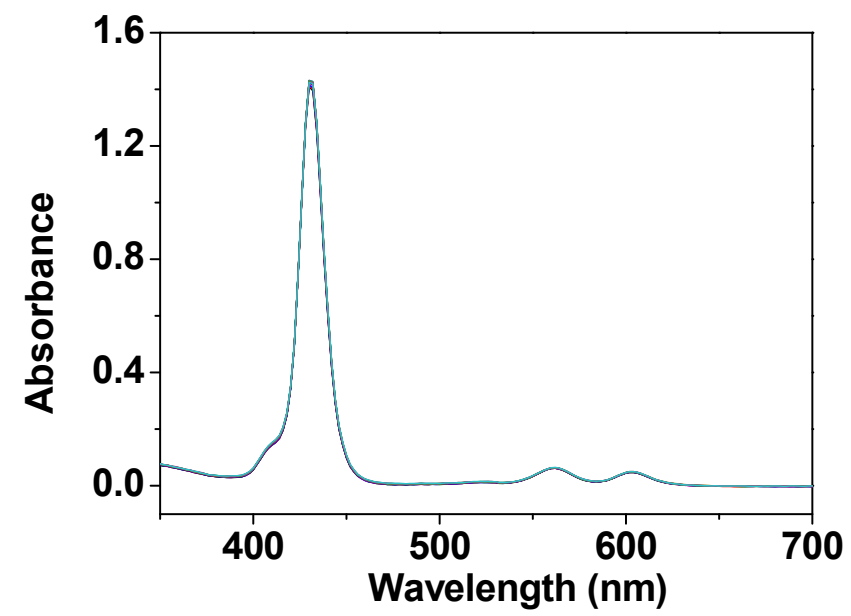

Figure S33. Titration of $\mathbf{Z n - 1}(0.5 \mu \mathrm{M})$ with substrate $\mathbf{1 f}$ in $\mathrm{CH}_{3} \mathrm{CN}$ : DMF (3:1 in volume) as monitored by UV-Vis $\left(25^{\circ} \mathrm{C}\right)$.

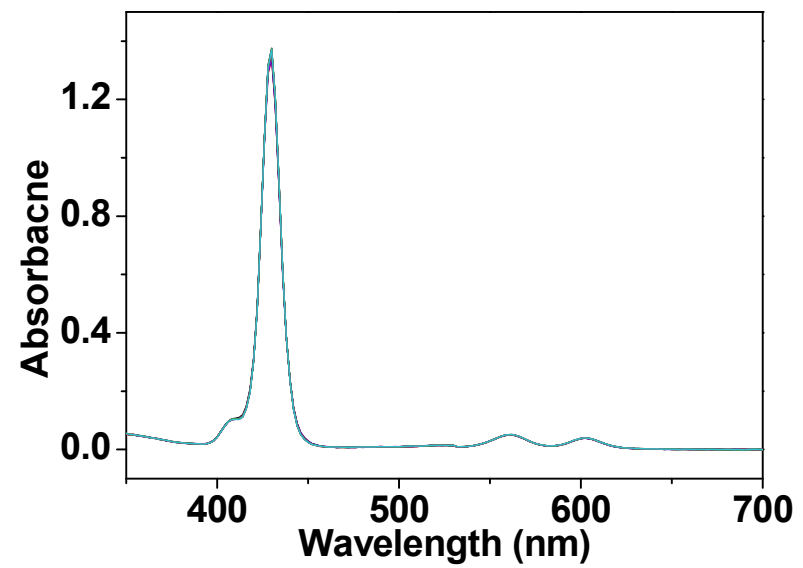

Figure S34. Titration of $\mathbf{Z n - 2}(0.5 \mu \mathrm{M})$ with substrate $\mathbf{1 f}$ in $\mathrm{CH}_{3} \mathrm{CN}$ : DMF (3:1 in volume) as monitored by UV-Vis $\left(25^{\circ} \mathrm{C}\right)$.

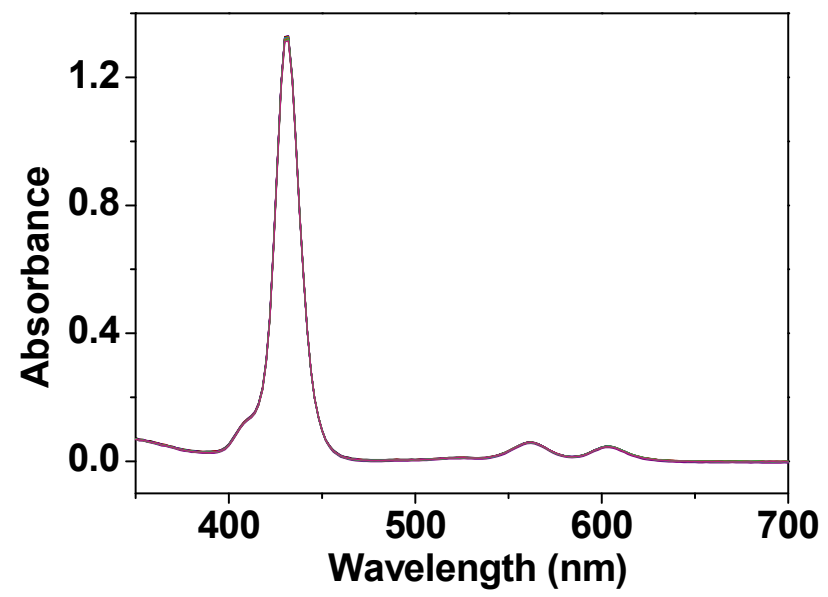

Figure S35. Titration of $\mathbf{Z n - 1}(0.5 \mu \mathrm{M})$ with substrate $\mathbf{1 g}$ in $\mathrm{CH}_{3} \mathrm{CN}$ : DMF (3:1 in volume) as monitored by UV-Vis $\left(25^{\circ} \mathrm{C}\right)$. 


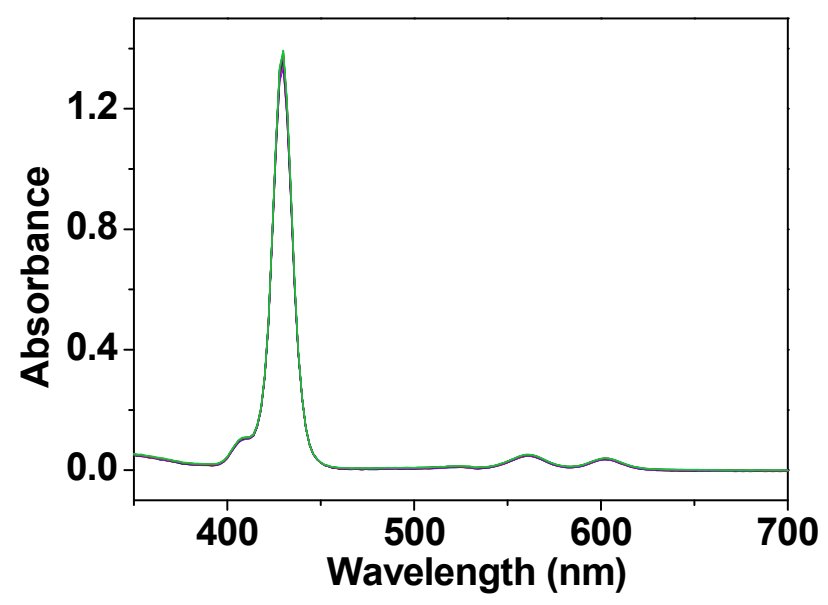

Figure S36. Titration of $\mathbf{Z n - 2}(0.5 \mu \mathrm{M})$ with substrate $\mathbf{1 g}$ in $\mathrm{CH}_{3} \mathrm{CN}$ : DMF (3:1 in volume) as monitored by UV-Vis $\left(25^{\circ} \mathrm{C}\right)$.

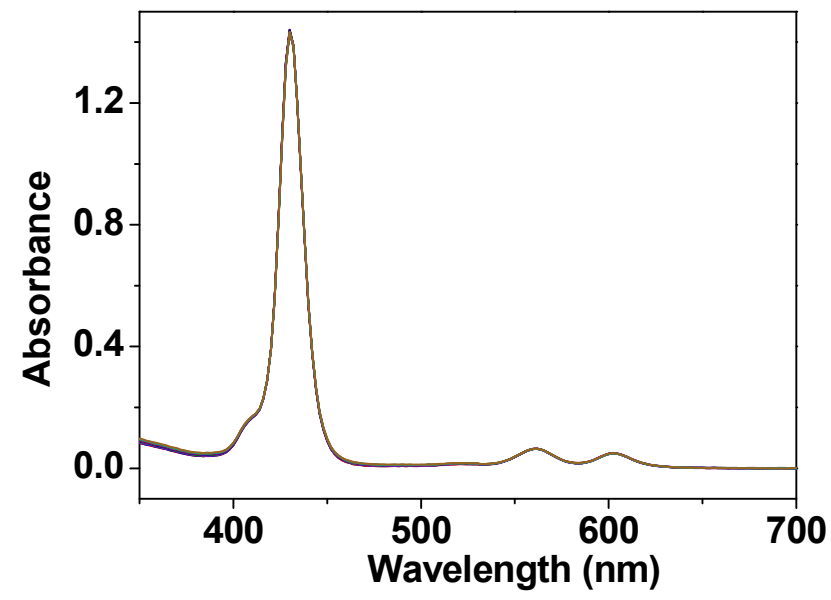

Figure S37. Titration of $\mathbf{Z n - 1}(0.5 \mu \mathrm{M})$ with substrate $\mathbf{3 f}$ in $\mathrm{CH}_{3} \mathrm{CN}$ : DMF (3:1 in volume) as monitored by UV-Vis $\left(25^{\circ} \mathrm{C}\right)$.

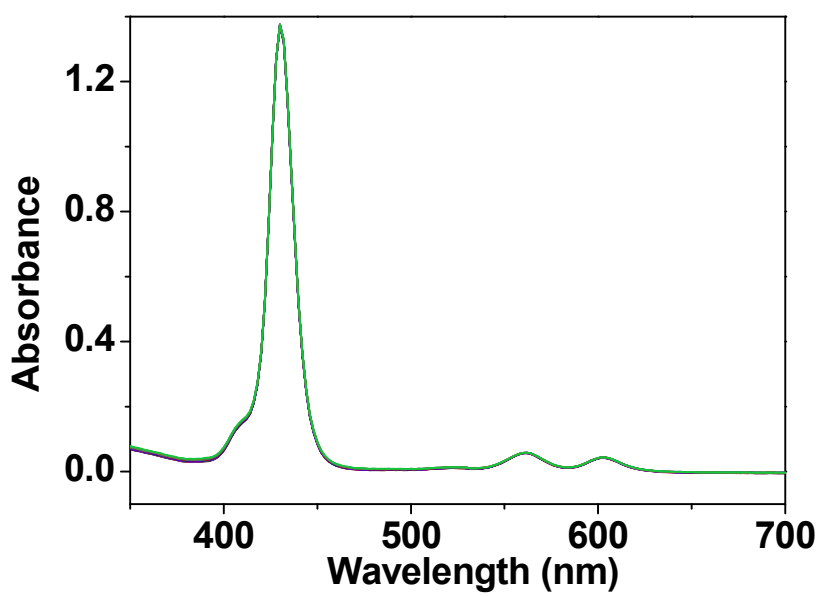

Figure S38. Titration of $\mathbf{Z n - 1}(0.5 \mu \mathrm{M})$ with substrate $\mathbf{3 g}$ in $\mathrm{CH}_{3} \mathrm{CN}$ : DMF (3:1 in volume) as monitored by UV-Vis $\left(25^{\circ} \mathrm{C}\right)$. 


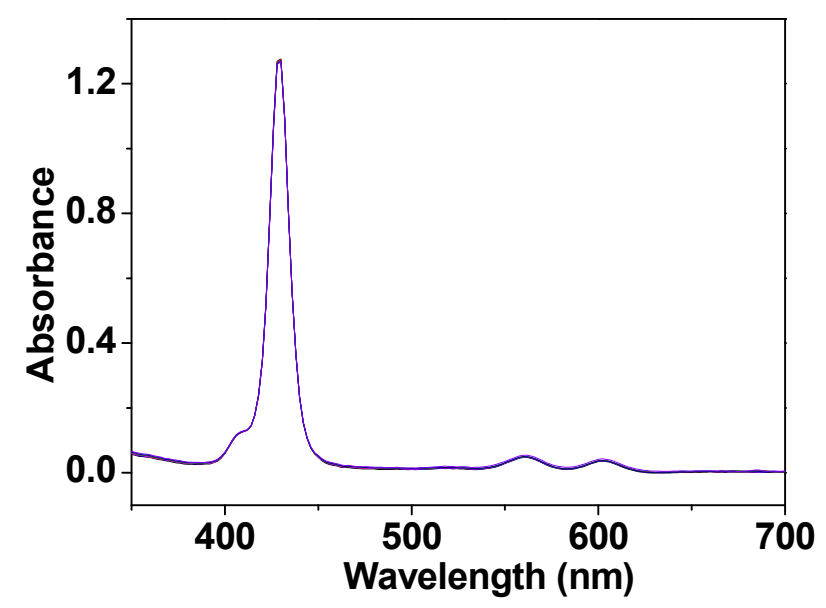

Figure S39. Titration of $\mathbf{Z n - 2}(0.5 \mu \mathrm{M})$ with substrate $\mathbf{3 f}$ in $\mathrm{CH}_{3} \mathrm{CN}$ : DMF (3:1 in volume) as monitored by UV-Vis $\left(25^{\circ} \mathrm{C}\right)$.

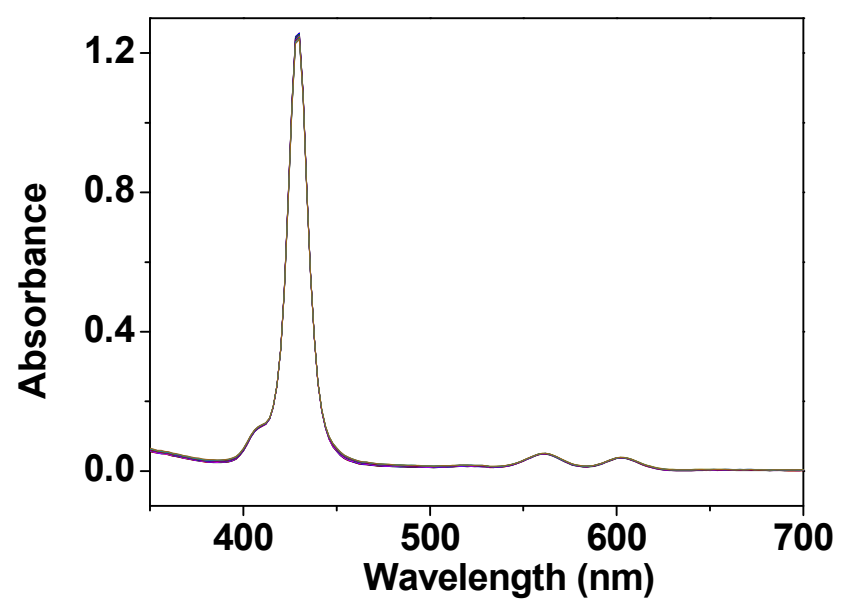

Figure S40. Titration of $\mathbf{Z n - 2}(0.5 \mu \mathrm{M})$ with substrate $\mathbf{3 g}$ in $\mathrm{CH}_{3} \mathrm{CN}$ : DMF (3:1 in volume) as monitored by UV-Vis $\left(25^{\circ} \mathrm{C}\right)$.
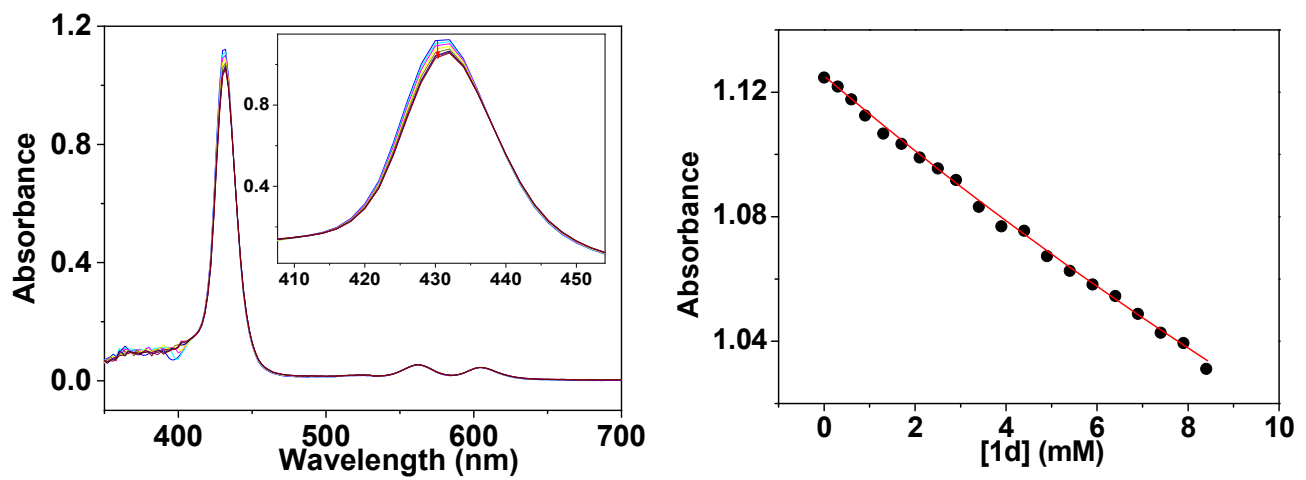

Figure S41. UV-Vis titration of $\mathbf{1 d}$ in $\mathrm{CH}_{3} \mathrm{CN}$ : DMF (3:1 in volume). (a) Titration of $\mathbf{Z n - 1}$ (0.5 $\mu \mathrm{M})$ with $\mathbf{1 d}$ as monitored by UV-Vis. (b) Binding isotherm from UV-Vis analysis at $430 \mathrm{~nm}\left(K_{a}=\right.$ 
$17 \pm 5 \mathrm{M}^{-1}$ at $\left.25^{\circ} \mathrm{C}\right)$.
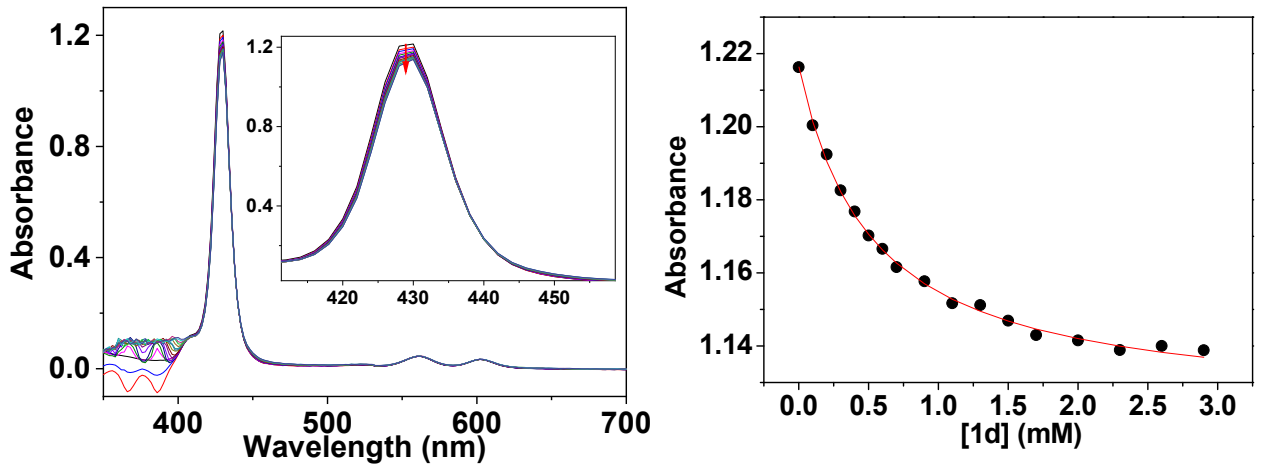

Figure S42. UV-Vis titration of $\mathbf{1 d}$ in $\mathrm{CH}_{3} \mathrm{CN}$ : DMF (3:1 in volume). (a) Titration of $\mathbf{Z n - 2}$ (0.5 $\mu \mathrm{M})$ with $1 \mathrm{~d}$ as monitored by UV-Vis. (b) Binding isotherm from UV-Vis analysis at $428 \mathrm{~nm}$. ( $K_{a}$ $=1923 \pm 103 \mathrm{M}^{-1}$ at $25^{\circ} \mathrm{C}$ ).
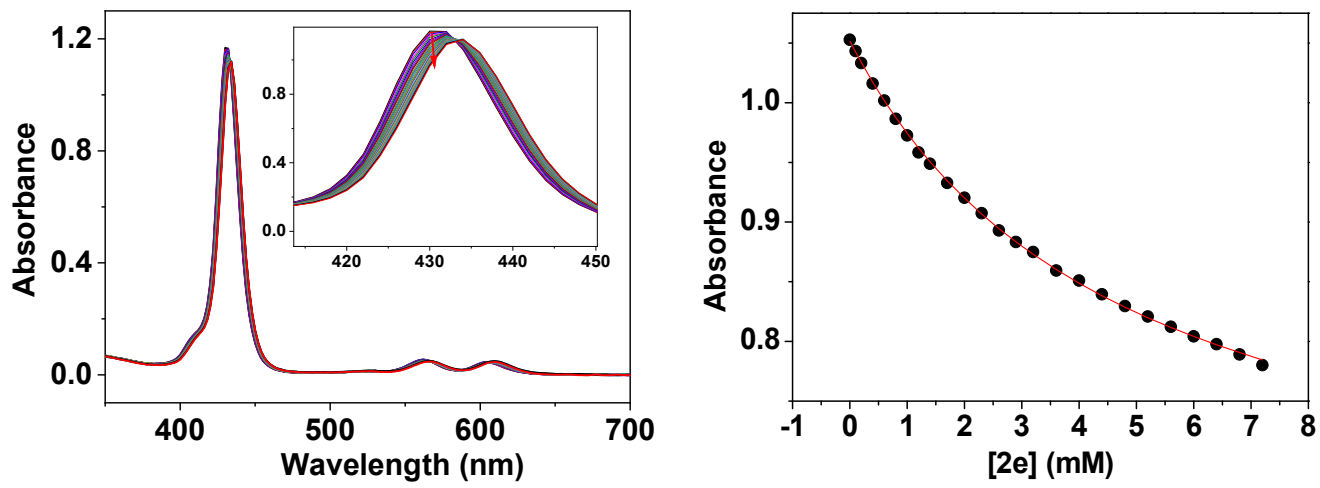

Figure S43. UV-Vis titration of $2 \mathbf{e}$ in $\mathrm{CH}_{3} \mathrm{CN}$ : DMF (3:1 in volume). (a) Titration of $\mathbf{Z n - 1}$ ( 0.5 $\mu \mathrm{M})$ with $2 \mathrm{e}$ as monitored by UV-Vis. (b) Binding isotherm from UV-Vis analysis at $430 \mathrm{~nm}\left(K_{a}=\right.$ $212 \pm 5 \mathrm{M}^{-1}$ at $25^{\circ} \mathrm{C}$ ).
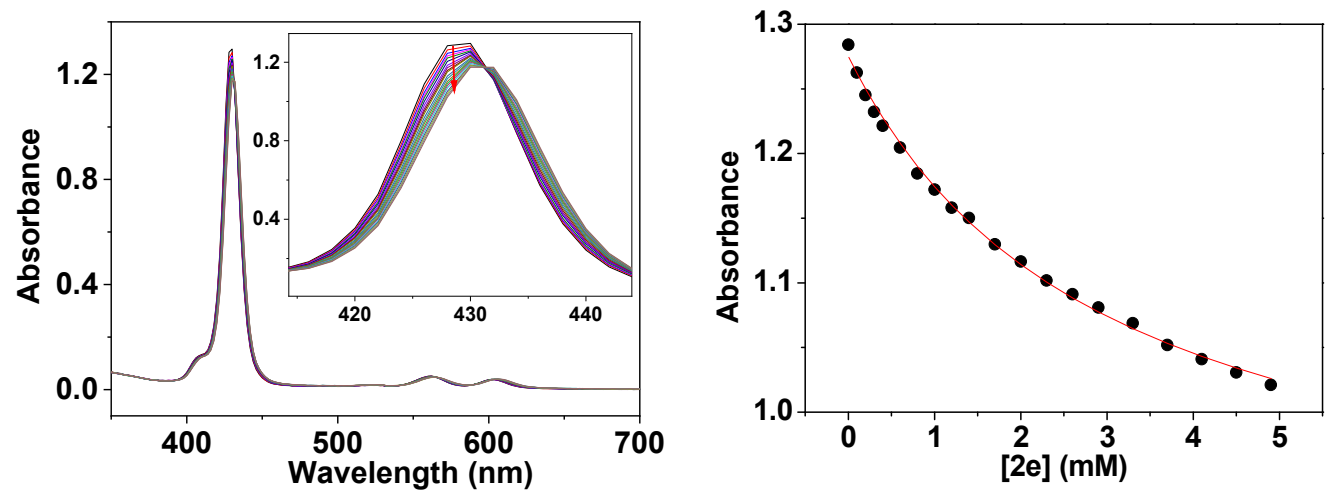

Figure S44. UV-Vis titration of $2 \mathbf{e}$ in $\mathrm{CH}_{3} \mathrm{CN}$ : DMF (3:1 in volume). (a) Titration of $\mathbf{Z n - 2}$ (0.5 $\mu \mathrm{M})$ with $2 \mathrm{e}$ as monitored by UV-Vis. (b) Binding isotherm from UV-Vis analysis at $428 \mathrm{~nm}\left(K_{a}=\right.$ $333 \pm 25 \mathrm{M}^{-1}$ at $\left.25^{\circ} \mathrm{C}\right)$. 


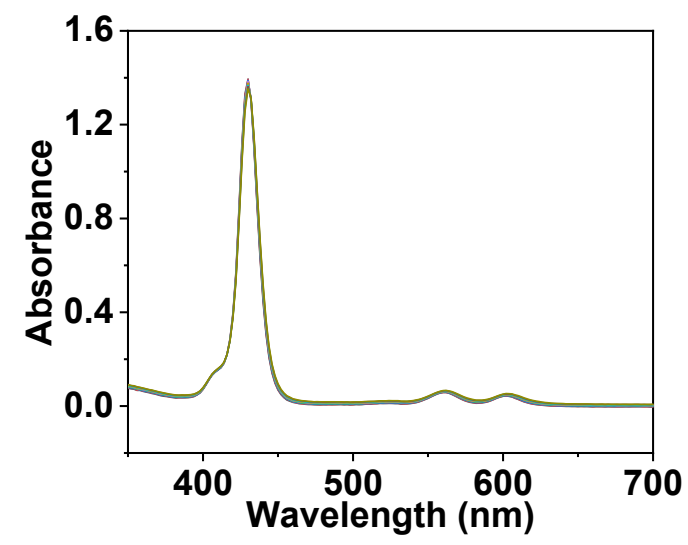

Figure S45. Titration of $\mathbf{Z n - 1}(0.5 \mu \mathrm{M})$ with substrate $\mathbf{3 e}$ in $\mathrm{CH}_{3} \mathrm{CN}$ : DMF (3:1 in volume) as monitored by UV-Vis $\left(25^{\circ} \mathrm{C}\right)$.
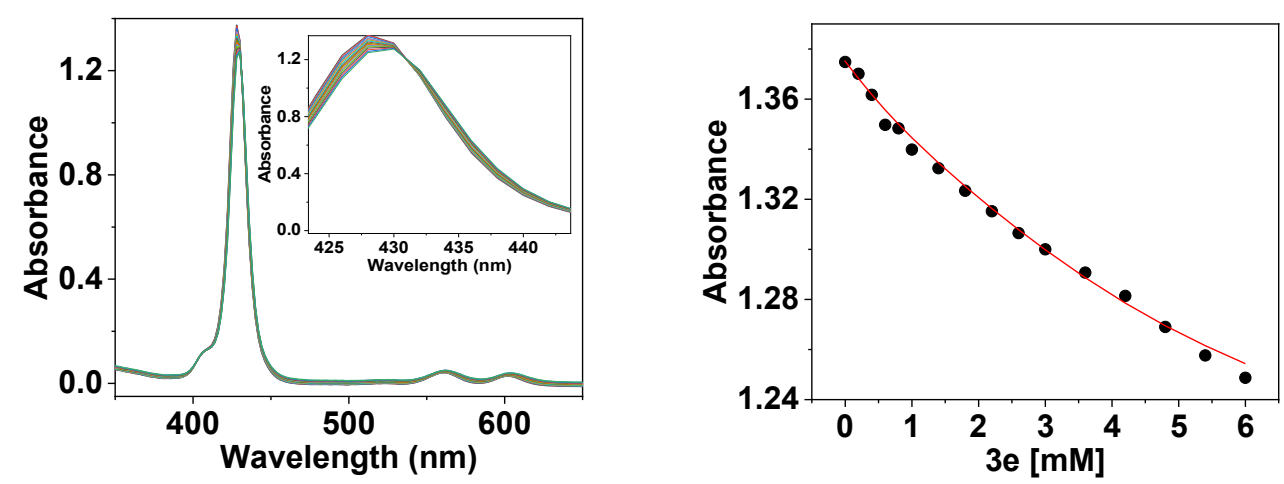

Figure S46. UV-Vis titration of $\mathbf{3 e}$ in $\mathrm{CH}_{3} \mathrm{CN}$ : DMF (3:1 in volume). (a) Titration of $\mathbf{Z n - 2}(0.5$ $\mu \mathrm{M})$ with 3e as monitored by UV-Vis. (b) Binding isotherm from UV-Vis analysis at $428 \mathrm{~nm}\left(K_{a}=\right.$ $94 \pm 18 \mathrm{M}^{-1}$ at $\left.25^{\circ} \mathrm{C}\right)$.

2.5 Characterization of catalytic products in the Acyl-transfer reaction.

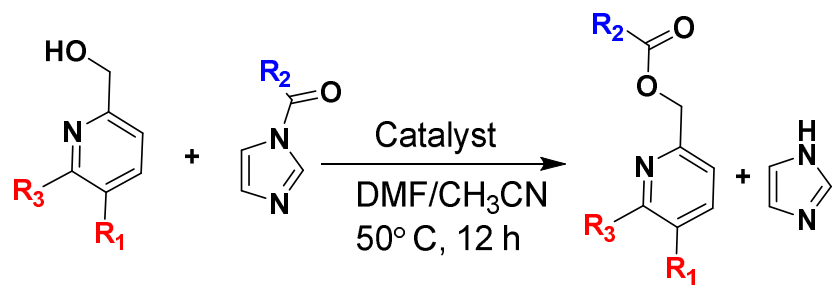<smiles>CC(=O)OCc1ccncc1</smiles>

Acetic acid pyridin-4-ylmethyl ester:

${ }^{1}$ H NMR (400 MHz, CDCl $): \delta=8.60$ (2H, s), 7.25 (2H, d, J=4 Hz), $5.13(2 \mathrm{H}, \mathrm{s})$, $2.16(3 \mathrm{H}, \mathrm{s})$. 


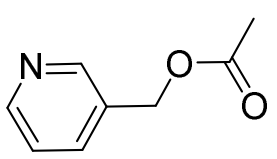

3-AMPy

Acetic acid pyridin-3-ylmethyl ester:

${ }^{1} \mathbf{H}$ NMR (400 MHz, CDCl $): \delta=8.63(1 \mathrm{H}, \mathrm{s}), 8.58(1 \mathrm{H}, \mathrm{d}, \mathrm{J}=4 \mathrm{~Hz}), 7.70(1 \mathrm{H}, \mathrm{d}, \mathrm{J}=8$ Hz), $7.30(1 \mathrm{H}, \mathrm{q}, \mathrm{J}=2.8,4,6.8 \mathrm{~Hz}), 5.13(2 \mathrm{H}, \mathrm{s}), 2.11(3 \mathrm{H}, \mathrm{s})$.

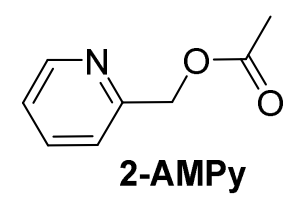

Acetic acid pyridin-2-ylmethyl ester:

${ }^{1}$ H NMR (400 MHz, $\left.\mathbf{C D C l}_{3}\right): \delta=8.61(1 \mathrm{H}, \mathrm{s}), 7.71(1 \mathrm{H}, \mathrm{t}, \mathrm{J}=8 \mathrm{~Hz}), 7.37(1 \mathrm{H}, \mathrm{d}$, $\mathrm{J}=6.4 \mathrm{~Hz}), 7.24(1 \mathrm{H}, \mathrm{d}, \mathrm{J}=4 \mathrm{~Hz}), 5.23(2 \mathrm{H}, \mathrm{s}), 2.17(3 \mathrm{H}, \mathrm{s})$.

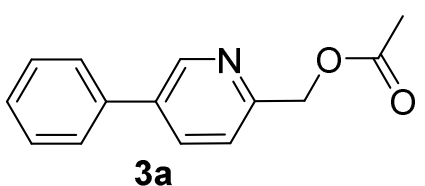

Acetic acid 5-phenyl-pyridin-2-ylmethyl ester (3a):

${ }^{1}$ H NMR (400 MHz, CDCl3): $\delta=8.83(1 \mathrm{H}, \mathrm{d}, \mathrm{J}=2.4 \mathrm{~Hz}), 7.90(1 \mathrm{H}, \mathrm{dd}, \mathrm{J}=4,12 \mathrm{~Hz})$, 7.59-7.57 (2H, m), 7.51-7.47 (2H, m), 7.44-7.41 (2H, m), $5.27(2 \mathrm{H}, \mathrm{s}), 2.18(3 \mathrm{H}, \mathrm{s})$. ${ }^{13}$ C NMR (400 MHz, CDCl 3$): ~ \delta=170.76,154.45,148.04,137.47,136.01,135.16$, $129.14,128.23,127.17,121.95,66.77,20.97$.

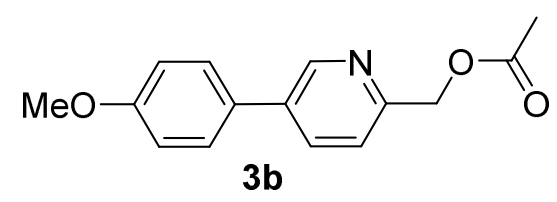

Acetic acid 5-(4-methoxy-phenyl)-pyridin-2-ylmethyl ester (3b):

${ }^{1}$ H NMR (400 MHz, CDCl 3$): \delta=8.79(1 \mathrm{H}, \mathrm{d}, \mathrm{J}=2 \mathrm{~Hz}), 7.85(1 \mathrm{H}, \mathrm{dd}, \mathrm{J}=4,8 \mathrm{~Hz})$, 7.53-7.50 (2H, m), $7.40(1 \mathrm{H}, \mathrm{d}, \mathrm{J}=8 \mathrm{~Hz}), 7.02-7.00(2 \mathrm{H}, \mathrm{m}), 5.25(2 \mathrm{H}, \mathrm{s}), 3.86(3 \mathrm{H}, \mathrm{s})$, $2.18(3 \mathrm{H}, \mathrm{s})$.

${ }^{13}$ C NMR (400 MHz, CDCl3): $\delta=170.77,159.86,153.73,147.67,135.64,134.63$, $129.85,128.24,122.00,114.61,66.82,55.40,20.97$ 


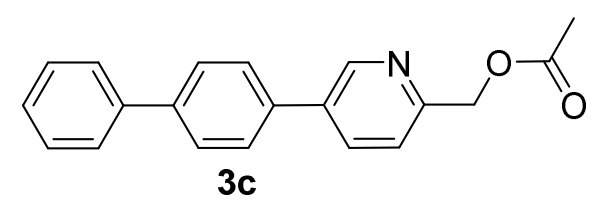

Acetic acid 5-biphenyl-4-yl-pyridin-2-ylmethyl ester (3c):

${ }^{1} \mathbf{H}$ NMR (400 MHz, CDCl 3$): ~ \delta=8.88(1 \mathrm{H}, \mathrm{d}, \mathrm{J}=4 \mathrm{~Hz}), 7.94(1 \mathrm{H}, \mathrm{dd}, \mathrm{J}=2.4,8 \mathrm{~Hz})$, 7.73-7.70 (2H, m), 7.67-7.63 (4H, m), 7.49-7.44 (3H, m), 7.40-7.36 (1H, m), 5.29 (2H, s), $2.19(3 \mathrm{H}, \mathrm{s})$.

${ }^{13}$ C NMR (400 MHz, CDCl3): $\delta=169.72,153.48,146.89,140.13,139.31,135.26$, $134.49,133.96,127.88,126.83,126.61,126.47,126.05,120.97,65.74,19.94$.

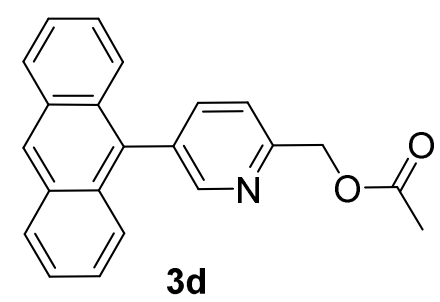

Acetic acid 5-anthracen-9-yl-pyridin-2-ylmethyl ester (3d):

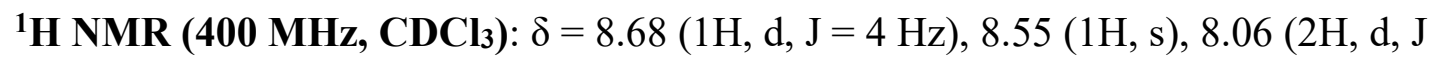
$=8.0 \mathrm{~Hz}), 7.81(1 \mathrm{H}, \mathrm{dd}, \mathrm{J}=2.0,8.0 \mathrm{~Hz}), 7.62-7.58(3 \mathrm{H}, \mathrm{m}), 7.51-7.41(2 \mathrm{H}, \mathrm{m}), 7.41-$ $7.37(2 \mathrm{H}, \mathrm{m}), 5.42(2 \mathrm{H}, \mathrm{s}), 2.26(3 \mathrm{H}, \mathrm{s})$.

${ }^{13}$ C NMR (400 MHz, CDCl 3$): ~ \delta=170.82,154.99,151.46,139.64,133.88,131.30$, $130.50,127.67,126.01,125.31,121.55,66.88,21.04$.<smiles>CC(=O)OCc1cccc(C)n1</smiles>

Acetic acid 6-methyl-pyridin-2-ylmethyl ester (3f):

${ }^{1}$ H NMR (400 MHz, CDCl3): $\delta=7.59(1 \mathrm{H}, \mathrm{t}, \mathrm{J}=8 \mathrm{~Hz}), 7.15(1 \mathrm{H}, \mathrm{d}, \mathrm{J}=8 \mathrm{~Hz}), 7.10$ $(1 \mathrm{H}, \mathrm{d}, \mathrm{J}=8 \mathrm{~Hz}), 5.18(2 \mathrm{H}, \mathrm{s}), 2.56(3 \mathrm{H}, \mathrm{s}), 2.15(3 \mathrm{H}, \mathrm{s})$.

${ }^{13}$ C NMR (400 MHz, CDCl 3$): ~ \delta=170.68,158.32,155.05,136.95,122.50,118.84$, $67.08,24.33,20.94$. 
<smiles>CC(=O)OCc1cccc(-c2ccccc2)n1</smiles>

$3 g$

Acetic acid 6-phenyl-pyridin-2-ylmethyl ester (3g):

${ }^{1} \mathbf{H}$ NMR (400 MHz, CDCl $): \delta=8.01-7.99(2 \mathrm{H}, \mathrm{m}), 7.77(1 \mathrm{H}, \mathrm{t}, \mathrm{J}=8 \mathrm{~Hz}), 7.66(1 \mathrm{H}$, $\mathrm{d}, \mathrm{J}=8 \mathrm{~Hz}), 7.50-7.46(2 \mathrm{H}, \mathrm{m}), 7.44-7.41(1 \mathrm{H}, \mathrm{m}), 7.30(1 \mathrm{H}, \mathrm{d}, \mathrm{J}=8 \mathrm{~Hz}), 5.31(2 \mathrm{H}$, s), $2.19(3 \mathrm{H}, \mathrm{s})$.

${ }^{13}$ C NMR (400 MHz, CDCl $): \delta=169.71,156.15,154.76,138.08,136.40,127.72$, $125.99,118.80,118.55,66.09,28.68,19.97$.<smiles>CC(C)CC(=O)OCc1ccccn1</smiles>

3-Methyl-butyric acid pyridin-2-ylmethyl ester (3e):

${ }^{1}$ H NMR (400 MHz, CDCl $): \delta=8.60(1 \mathrm{H}, \mathrm{d}, \mathrm{J}=4 \mathrm{~Hz}), 7.70(1 \mathrm{H}, \mathrm{ddd}, \mathrm{J}=2.0,8.0$ Hz), $7.36(1 \mathrm{H}, \mathrm{d}, \mathrm{J}=8 \mathrm{~Hz}), 7.24-7.21(1 \mathrm{H}, \mathrm{m}), 5.23(2 \mathrm{H}, \mathrm{s}), 2.30(2 \mathrm{H}, \mathrm{d}, \mathrm{J}=8 \mathrm{~Hz})$, 2.21-2.11 (1H, m), $0.98(6 \mathrm{H}, \mathrm{d}, \mathrm{J}=8 \mathrm{~Hz})$.

${ }^{13}$ C NMR (400 MHz, CDCl3): $\delta=172.74,156.04,149.47,136.72,122.80,121.77$, $66.61,43.28,25.71,22.42$.

\section{Kinetic Analysis of the Acyl-transfer Reaction}

Table S3. The reaction rate at various concentration of substrates in the presence of Zn-1 and Zn-2.

\begin{tabular}{c|c|c}
\hline Concentrations of substrates & $\begin{array}{c}\text { Reaction rate in } \mathbf{Z n - 1} \\
\left(\mathrm{mM} \cdot \mathrm{h}^{-1}\right)\end{array}$ & $\begin{array}{c}\text { Reaction rate in Zn-1 } \\
\left(\mathrm{mM} \cdot \mathrm{h}^{-1}\right)\end{array}$ \\
\hline 2-PC $(12 \mathrm{mM}), \mathrm{NAI}(12 \mathrm{mM})$ & 1.6 & 2.2 \\
\hline 2-PC $(12 \mathrm{mM}), \mathrm{NAI}(18 \mathrm{mM})$ & 3.0 & 3.2 \\
\hline 2-PC $(18 \mathrm{mM}), \mathrm{NAI}(12 \mathrm{mM})$ & 3.5 & 4.6 \\
\hline
\end{tabular}



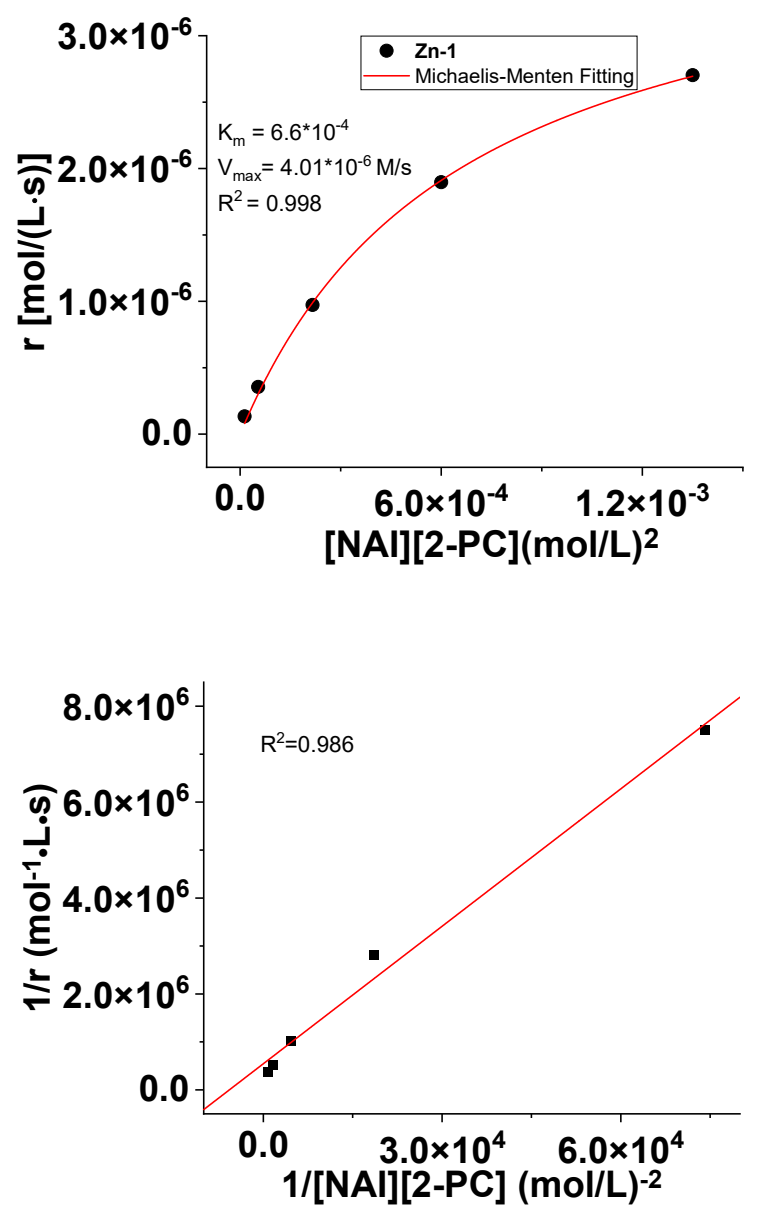

Figure S47. (a) Steady state kinetic analyses using the Michaelis Menten model and (b) Lineweaver Burk plot for Zn-1 (2-PC and NAI as the substrates).

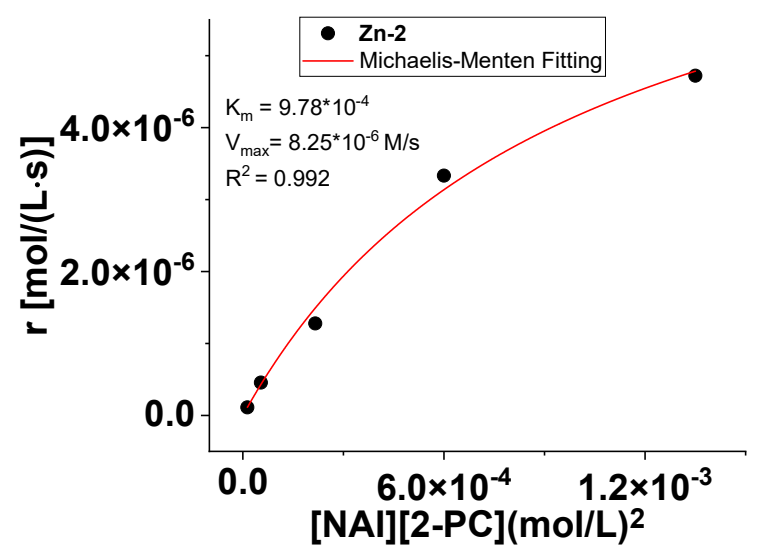




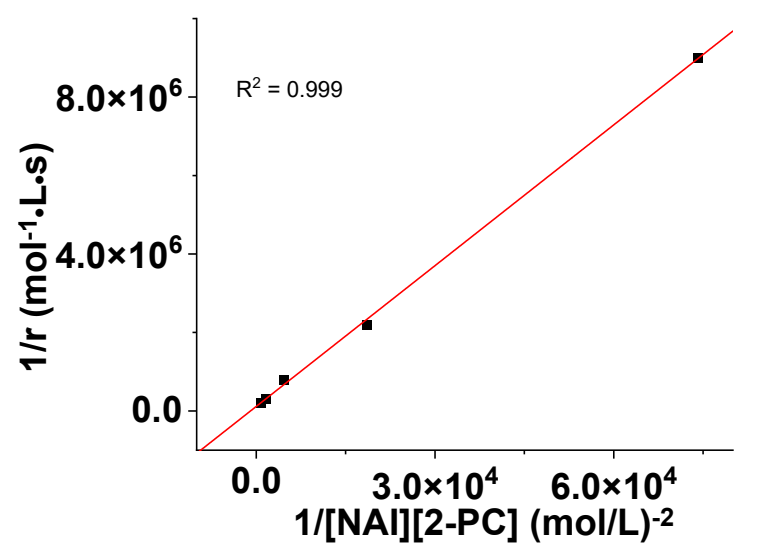

Figure S48. (a) Steady state kinetic analyses using the Michaelis Menten model and (b) Lineweaver Burk plot for Zn-2 (2-PC and NAI as the substrates).

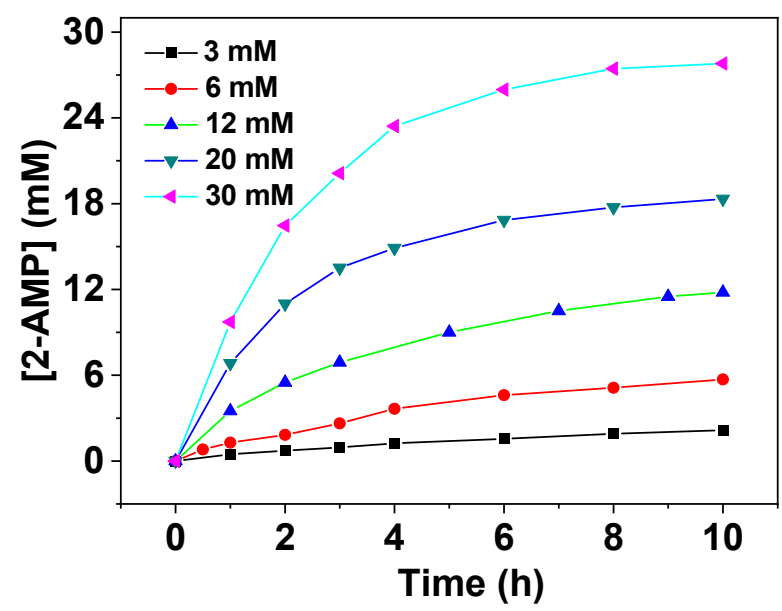

Figure S49. Plots of kinetic results with varying concentration of substrates in present of Zn-1. Black squares: [2-PC] $=4.5 \mathrm{mM}$, [NAI] $=3 \mathrm{mM}$; red circles: [2-PC] $=9 \mathrm{mM}$, $[\mathrm{NAI}]=6 \mathrm{mM}$; blue triangles: [2-PC] $=18 \mathrm{mM},[\mathrm{NAI}]=12 \mathrm{mM}$; green triangle: [2-PC] $=30 \mathrm{mM},[\mathrm{NAI}]=20 \mathrm{mM}$; pink triangles: $[2-\mathrm{PC}]=45 \mathrm{mM}$, [NAI] $=30 \mathrm{mM}$.

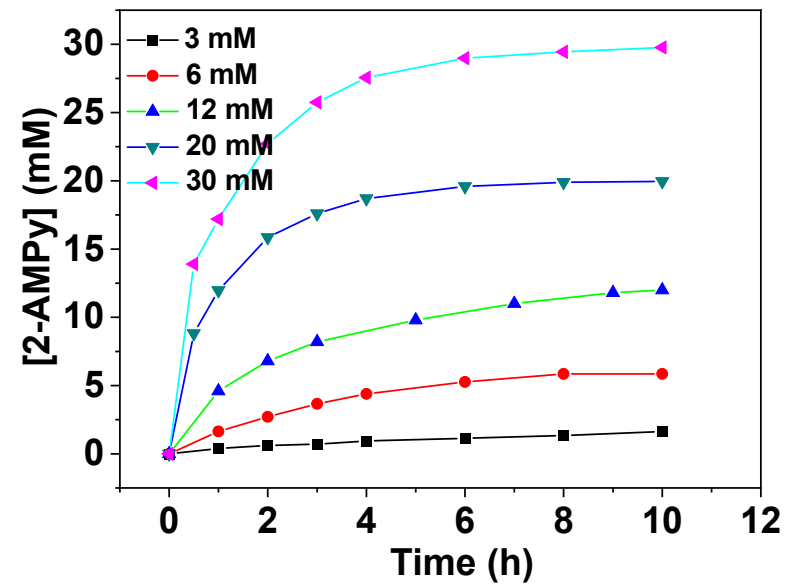

Figure S50. Plots of kinetic results with varying concentration of substrates in present of Zn-2. Black squares: [2-PC] $=4.5 \mathrm{mM}$, [NAI] $=3 \mathrm{mM}$; red circles: [2-PC] $=9 \mathrm{mM}$, 
$[\mathrm{NAI}]=6 \mathrm{mM}$; blue triangles: $[2-\mathrm{PC}]=18 \mathrm{mM},[\mathrm{NAI}]=12 \mathrm{mM}$; green triangles: [2$\mathrm{PC}]=30 \mathrm{mM},[\mathrm{NAI}]=20 \mathrm{mM}$; pink triangles: $[2-\mathrm{PC}]=45 \mathrm{mM},[\mathrm{NAI}]=30 \mathrm{mM}$.
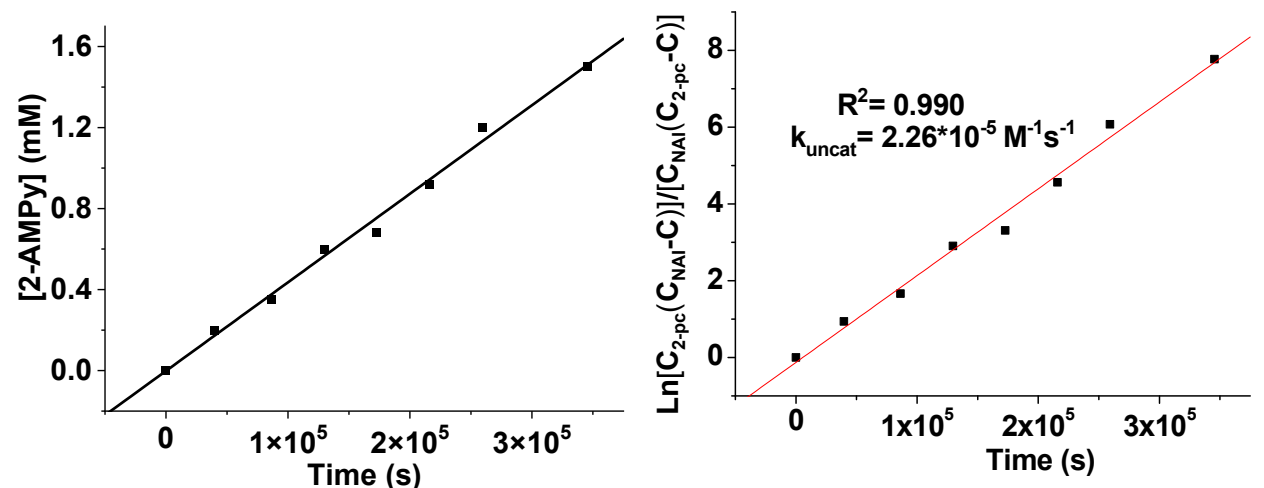

Figure S51. Kinetic data for the background reaction (no catalyst present).

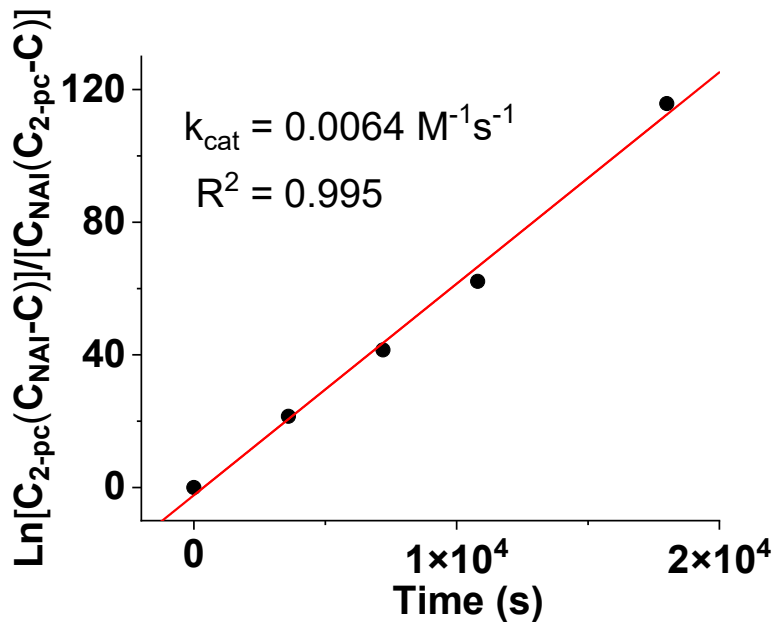

Figure S52. Rates of catalysed reactions in present of Zn-1: $k_{\text {cat }} / k_{\text {uncat }}=283$.

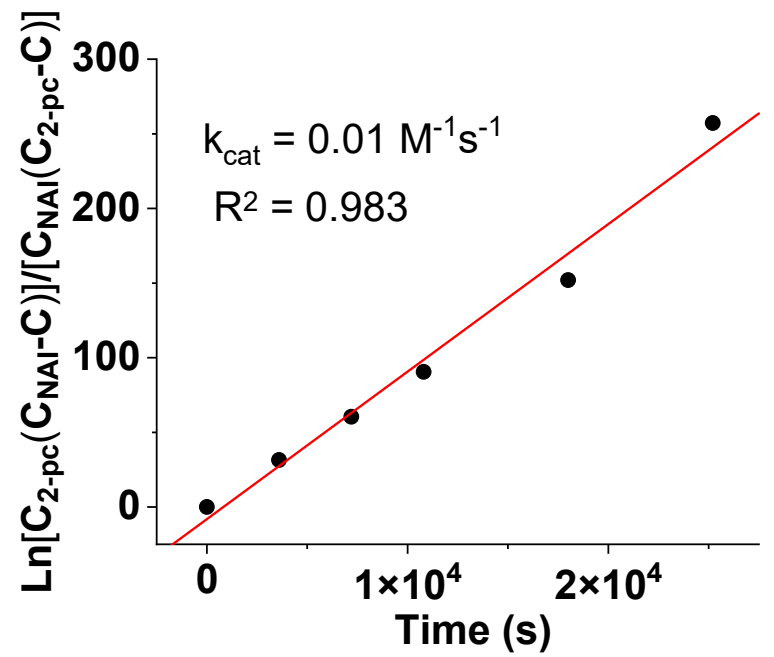

Figure S53. Rates of catalysed reactions in present of Zn-2: $k_{\text {cat }} / k_{\text {uncat }}=442$. 
Table S4. Control experiments of acyl transfer reaction.

\begin{tabular}{|c|c|c|c|c|}
\hline Entry & \multicolumn{2}{|c|}{ Catalyst } & Yield $(\%)$ & Initial Rate $\left(\mathrm{mM} \cdot \mathrm{h}^{-1}\right)$ \\
\hline 1 & \multicolumn{2}{|c|}{$\mathrm{Zn}-1(4 \mathrm{~mol} \%)^{\mathrm{a}}$} & 99.0 & 3.5 \\
\hline 2 & \multicolumn{2}{|c|}{$\mathrm{Zn}-1(4 \mathrm{~mol} \%)+$ Imidazole $(4 \mathrm{~mol} \%)^{a}$} & 70 & 2.4 \\
\hline 3 & \multicolumn{2}{|c|}{ Zn-TAPPa } & 6.8 & 0.08 \\
\hline 4 & \multicolumn{2}{|c|}{$\mathrm{Zn}-2(4 \mathrm{~mol} \%)^{\mathrm{a}}$} & 99.5 & 4.6 \\
\hline 5 & \multicolumn{2}{|c|}{$\mathrm{Zn}-2(4 \mathrm{~mol} \%)+$ Imidazole $(4 \mathrm{~mol} \%)^{a}$} & 70 & 2.2 \\
\hline 6 & \multicolumn{2}{|c|}{$\mathrm{L}^{\mathrm{a}}$} & 4.8 & 0.05 \\
\hline 7 & \multicolumn{2}{|c|}{ No catalyst ${ }^{b}$} & 12.5 & 0.02 \\
\hline
\end{tabular}

a Reaction condition: [2-PC] $=18 \mathrm{mM},[\mathrm{NAI}]=12 \mathrm{mM}$, in $\mathrm{CH}_{3} \mathrm{CN}$ : DMF (3:1 in volume), at $50^{\circ} \mathrm{C}$ for $12 \mathrm{~h}$.

beaction condition: [2-PC] $=18 \mathrm{mM},[\mathrm{NAI}]=12 \mathrm{mM}$, in $\mathrm{CH}_{3} \mathrm{CN}$ : DMF (3:1 in volume), at $50^{\circ} \mathrm{C}$ for $96 \mathrm{~h}$.

\section{Supplemental References}

1. W.-M. Meng, B. Breiner, K. Rissanen, J. D. Thoburn, J. K. Clegg and J. R. Nitschke, A self-assembled $\mathrm{M}_{8} \mathrm{~L}_{6}$ cubic cage that selectively encapsulates large aromatic guests. Angrew. Chem. Int. Ed. 2011, 50, 3479-3483.

2. S.-T. Bai, C. B. Bheeter and J. N. H. Reek, Hydrogen bond directed ortho-selective C-H borylation of secondary aromatic amides. Angrew. Chem. Int. Ed. 2019, 58, 13039-13043.

3. G.-H. Chan, D. Y. Ong, Z. H. Yen and S. Chiba, Reduction of N,Ndimethylcarboxamides to aldehydes by sodium hydride-iodide composite. Helv. Chim. Acta 2018, 101, e1800049.

4. D. Y. Ong, Z. H. Yen, A. Yoshii, J. R. Imbernon, R. Takita and S. Chiba, Controlled reduction of carboxamides to alcohols or amines by zinc hydrides. Angew. Chem. Int. Ed. 2019, 58, 4992-4997.

5. B. H. Patel and A. G. M. Barrett, Total synthesis of resorcinol amide Hsp90 inhibitor AT13387. J. Org. Chem. 2012, 77, 11296-11301.

6. P. Kuzmič, Program DYNAFIT for the analysis of enzyme kinetic data: application to HIV proteinase. Anal. Biochem. 1996, 237, 260-273. 